\title{
EL TABACO COMO RETO EDUCATIVO. UNA REVISIÓN DESDE UNA PERSPECTIVA SOCIOPEDAGÓGICA
}

Juan Agustín Morón Marchena

Universidad de Sevilla

"Un cigarrillo es un producto manufacturado hábilmente para liberar exactamente la dosis de nicotina necesaria para mantener al usuario adicto el mayor tiempo posible antes de matarlo»

Gro Harlem Brudtland, Directora General de la OMS (Organización Mundial de la Salud)

Muchas de las personas que nos rodean fuman. Aunque cada vez existe más conciencia de considerar el tabaco como "perjudicial" -para algunos es ¿la adicción más perniciosa en el mundo desarrollado? (Solano Reina et al., 2000)-, el debate sobre el tema está abierto, en las aulas, en la "calle", en la vida cotidiana, en los medios de comunicación. Dudas y preguntas que se plantean a menudo (es o no es una droga, el dilema droga dura/blanda, legal o no), hechos preocupantes (el primer contacto a una edad cada vez más temprana, el mayor porcentaje de mujeres que se hacen fumadoras, etc.), complicado aún más cuando además entra en juego un componente ético, personal, la libertad al decidir si fumar o no.

En Congresos y reuniones científicas sobre Educación para la Salud en los que he participado para abordar la cuestión del tabaco, se han producido agrios debates sobre algunos de estos aspectos, que en otros ámbitos de la 
Promoción de la Salud no se dan. Pretendo en este artículo analizar el entorno que rodea al tabaco, la situación actual desde diversas perspectivas, porque la complejidad del mismo no puede entenderse sin comprender el marco global que le rodea (ya hecho en Morón Marchena, 1998a; 1999), más allá de una concepción didáctica de la que hay mucho escrito. El desarrollo histórico que ha hecho posible que en medio siglo esté difundido por todo el mundo, la legislación, la fiscalidad, los lobby de presión... nos sitúan ante una cuestión que debe abordarse desde una perspectiva socioeducativa, en la que no debe obviarse el elemento educativo ( $\mathrm{y}$ en muchos planteamientos se olvida), ni podemos caer en un "optimismo pedagógico" que considere que la solución al problema está en su tratamiento escolar.

\section{EL TABACO ¿UN RETO SOCIOPEDAGÓGICO?}

El tabaco, y toda la problemática que le rodea en el marco de la Educación para la Salud, no puede considerarse más que partiendo de un planteamiento de carácter sociopedagógico, por cuanto:

- Tiene efectos muy perjudiciales para la salud, pero debe abordarse desde una perspectiva social, superando la meramente sanitaria-médica. Conlleva aspectos económicos, fiscales, legislativos...

- La educación es el «arma» más importante para enfrentarse de manera preventiva al consumo del tabaco.

La prevención del tabaco es, pues, una tarea eminentemente social y comunitaria, y la educación es la herramienta primordial para abordar este tema. Delgado, Pablos y Sánchez (1996:32) dicen que es la droga enmascara$\mathrm{da}$, porque no se quiere percibir los daños que produce en la salud ni se tiene conciencia de la dependencia que ocasiona, idéntica a la de otras drogas. Es la principal responsable de toda una serie de enfermedades respiratorias y cardíacas, tiene que ver con los tipos más frecuentes de cáncer y últimamente con las enfermedades alérgicas. Posiblemente nada influye tan negativamente en este momento en la salud de los españoles como el tabaco. Para valorar el grado de dependencia, siguiendo a los citados autores, basta considerar que un $71 \%$ de los fumadores de más de un paquete y medio diario querría dejarlo y no lo consigue. Una parte importante no conseguirán nunca dejar de fumar si no se les ayuda a pasar el síndrome de abstinencia y se les capacita terapéuticamente en ciertas habilidades. Por otra parte, los jóvenes fuman en un porcentaje superior al de los adultos y, si la incidencia de enfermedades es tan elevada en los adultos con el actual nivel de tabaquismo, es lógico pensar que tales enfermedades se incrementarán, por lo que muchos no llegarán a la vejez por causa del tabaco. En España se han realizado muchas investigaciones sobre su consumo tanto en jóvenes como en adultos, revelando que se están alcanzando cada vez mayores proporciones y que ha llegado a ser la droga más consumida (Rooney y Villahoz, 1995:93). 
En las siguientes líneas nos acercaremos a la realidad del tabaco y su relación con la Educación para la Salud. Existen numerosos trabajos, investigaciones y publicaciones sobre el tabaco desde diversas perspectivas. Sin embargo, no es de los temas más tratados en el campo de la Promoción y Educación para la Salud. Hay que tener en cuenta que se convive con el tabaco, que está presente en cualquier lugar y momento; se dice que es una droga, pero tiene unas peculiaridades que requieren un abordaje peculiar. Al hablar del tabaco, debemos partir de la propia concepción holística y positiva de salud, considerando una serie de aspectos de índole social, referidos al contexto social, que en gran medida determinan a aquélla (González y García, 1998; De la Rosa Acosta, 1995). La salud es una cuestión global y no puede hablarse de ella en un contexto reducido, aunque existen aspectos que dependen de las condiciones concretas de un lugar determinado: la interrelación entre la salud y el entorno depende de "condiciones planetarias», como una tarea transdisciplinar. Por ello, el tabaco debe enfocarse de esta manera. Aunque se conocen sus nefastas consecuencias para la salud, parece imposible poder acabar con él, pues se interrelacionan numerosas variables que nos sitúan ante un problema ciertamente complejo. Pero de lo que no cabe duda es de que nos encontramos ante un tema que debe estar presente en el campo educativo. La Promoción y Educación para la Salud son fundamentales para eliminar $\rightarrow$ aminorar- y abordar los problemas del tabaco.

\section{ALGUNAS REFERENCIAS HISTÓRICAS SOBRE EL TABACO}

Hablar de los orígenes del tabaco nos lleva a medio siglo a.C., cuando Herodoto (484-425 a.C., al que Cicerón llamó Padre de la Historia) hace referencia a la costumbre de inhalar humo de plantas aromáticas. Desde entonces mucho se ha escrito sobre la procedencia y el descubrimiento del tabaco, sin que exista un acuerdo total, pero sí se sabe que la planta de la que se extrae proviene de las zonas costeras del continente americano y que los españoles fuimos los primeros europeos que la conocimos y trajimos (si bien otros testimonios fechan en el s. XIV su llegada a Europa).

Los descubridores españoles quedaron sorprendidos cuando los indios fumaban por la nariz unos cilindros de hojas enrolladas, a veces empleadas en ceremonias "religiosas", o cuando consumían hojas trituradas en cañas cortas o pipas, que a veces daba lugar a comportamientos extraños en sus consumidores (agitación, delirio). Los indígenas, que los llamaban de manera parecida a cohíba o cahoba, los ofrecían a los colonizadores a modo de presente. Algunos autores señalan que ya el 15 de octubre de 1492, Cristóbal Colón hacía referencia a las hojas secas que los indios les entregaban como dádiva (y a las que dio poco valor). Otros mantienen que el primer contacto con la extraña sustancia que los indios fumaban, inhalaban o consumían tuvo lugar algo más tarde, basándose también en el diario de Colón, concretamente el 6 de noviembre. 
Dos miembros de la tripulación de Colón, Luis de Torres y Rodrigo de Jerez fueron los primeros que vieron a los indios fumar tabaco en Gibara (Cuba) en noviembre de dicho año. Según la tradición, éste último -natural de la localidad onubense de Ayamonte-, fue el primer fumador europeo y el que introdujo la costumbre (Becoña, Galego y Lorenzo, 1988). Como una de las personas más significativas en el cultivo del tabaco en la península, quizás se le deba el término de «cigarro», al plantar las semillas en un cigarral, unos solares que en tiempos de verano eran habitualmente ocupados por las cigarras (o langostas africanas). También se dice que en 1528 el misionero Fray Romano Paré -acompañante de Cristóbal Colón en otro de sus viajesenvió al Rey Carlos V unas semillas para su cultivo, además de indicarle distintas virtudes medicinales (según otras fuentes, fray Ramón Pené envió a los Reyes en 1499 unas semillas para su cultivo). El tabaco llegó a España y de aquí a los países limítrofes. En Portugal se extendió rápidamente, donde el embajador francés Jean Nicot "investigó" las cualidades curativas del tabaco, cotejó los resultados con el médico del Rey de Portugal y se los remitió a la Reina de Francia, Catalina de Médicis, que promovió activamente su consumo en el país galo (se sitúa en el 1570 la entrada del tabaco en Francia). En Inglaterra se popularizó desde 1543 por sus propiedades curativas. La extensión fue rápida y continua. Los portugueses lo llevaron a África y Asia y los mercaderes judíos al resto de los países mediterráneos, llegando hasta Turquía.

A lo largo de la historia, se le ha conocido al tabaco con muy diversos nombres, relacionados normalmente con su actividad terapéutica (asociada sobre todo con las enfermedades y dolencias de origen frío, como resfriados, pulmonías..., por lo que se llamaba 'Herba Sancta' o 'Hierba Panacea') o por su uso entre personajes de alto nivel social ('Hierba del Embajador', 'Hierba de la Reina', 'Catalina Nuduca', 'Tetun', 'Hierba de Santa Cruz', 'Planta Medicea'). De la Champs promovió la denominación de 'nicotiana' a la planta de tabaco, en homenaje al embajador Nicot, aunque fue en 1753 cuando Linneo "oficializó" su nombre como 'Nicotiana Tabacum' en su libro Species Plantarum. No se sabe exactamente la procedencia del vocablo 'tabaco'. Para unos, proviene del nombre que los indígenas daban a la planta narcótica que hacían arder ('tabak'). Para Fernández Oviedo se debe al nombre del utensilio que los indios usaban para inhalar, llamado de manera semejante en las distintas islas, mientras que la planta o la sustancia recibía diferentes nombres según el sitio. Otros autores mantienen que su origen se debe a la isla Tabaco (Yucatán), en la que se descubrió la planta.

Mientras en nuestro país el consumo del tabaco se dio principalmente en las capas sociales de menor nivel, en las demás naciones europeas fue al revés, iniciándose en las capas de alto estatus social para posteriormente extenderse al resto. Pero no todo fueron facilidades para su extensión, pues desde pronto aparecieron detractores. En los siglos XVI y XVII se prohibió su consumo en público, publicándose incluso en ordenanzas reales y pontificias. De hecho, el Papa Urbano VIII decidió excomulgar en 1624 a los que 
fumasen en lugares próximos a las iglesias y sus alrededores. El Papa Inocencio XII también dictó una bula en 1690 manifestándose en contra de los consumidores de tabaco, con penas de excomunión (parece ser que algunos religiosos consumían de manera excesiva el tabaco en polvo).

Uno de los pioneros contrarios al tabaco fue Jacobo I de Inglaterra, que en 1604 prohibió su consumo público con distintas penas. En Alemania se dictó pena de muerte para los que fumaran, mascaran o inhalaran tabaco (1691) y en Prusia se prohibió fumar en público hasta 1848. A pesar de ello, en el s. XIX el tabaco era generalizado en toda Europa. Otras prohibiciones fueron expeditivas. En el s. XVII, el zar Miguel Federico de Rusia torturaba a los fumadores hasta que declararan quién les había suministrado el tabaco, para seguidamente cortarles a ambos la nariz y mandarlos a Siberia. El sultán Murad IV ordenó en el s. XVIII castigar a los fumadores con distintas medidas: mutilarles pies y/o manos, cortarles la cabeza, los brazos y las piernas. Amurates IV (Turquía) ordenó cortar la nariz y labios a los fumadores. El Sha de Persia también ordenaba cortar la nariz. En nuestro país, ya hemos dicho que era propio de las clases bajas y fue un vicio perseguido por la Santa Inquisición: curiosamente, el citado Rodrigo de Jerez fue encarcelado por la Inquisición por fumar.

En el primer cuarto del s. XVII se construyó la primera fábrica de tabaco en Sevilla (hasta entonces, los cigarrillos eran liados por los mismos consumidores, y el polvo se obtenía de modo artesanal). Posteriormente se hicieron otras por todo el territorio nacional, pero se cerraron para centralizar la producción en una nueva sede ampliada de Sevilla. La demanda era tal que en Cádiz se creó una factoría dedicada solamente a la producción de cigarros cuyos trabajadores eran mujeres. España fue el primer país en gravar fiscalmente la importación de tabaco a principios del s. XVII al decidir las Cortes que la Hacienda Pública se hiciera cargo de su comercialización. Así, en 1636 se creó en Castilla y León el primer monopolio del tabaco, favorecido por poseer España todas las tierras productoras. Al ir desintegrándose el Imperio, otras naciones europeas crearon sus propios monopolios.

La pipa común aparece en Inglaterra en el 1585 a raíz de la conquista de Virginia. Los españoles fuimos los inventores del papel de fumar. Aparecieron nuevos tipos de planta y de métodos de curado, y en la guerra de Crimea, entre 1854 y 1856 , se empezó a fumar envolviendo las hojas de tabaco picado en papel poroso. La primera herramienta para liar cigarrillos data de 1855 y diez años más tarde se inventó la máquina de hacer cigarros. En 1881 se patentó en los Estados Unidos la máquina de fabricar cigarrillos. En 1887, se concedió el monopolio del tabaco en nuestro país a una empresa privada, prorrogándose periódicamente hasta 1945, cuando se creó Tabacalera S.A. En 1962 el Banco de España se hizo con la mayoría de las acciones, hasta llegar a nuestros días, en plena época de privatización de empresas públicas, en la que Altadis la asumió (ahora, existe monopolio estatal en la distribución minorista con los estancos, y el resto de la distribución está liberalizada). 
Al final del s. XIX comenzó la industrialización del tabaco, expandiéndose rápidamente la elaboración del cigarrillo. Cabe destacar la American Tobacco Company, gran empresa estadounidense que en 1911 tuvo que dividirse en cuatro compañías más pequeñas debido a la ley antimonopolio. Por entonces, los hombres pedían permiso en las reuniones con mujeres, que no fumaban -excepto las prostitutas- ni tampoco los menores de 21 años. La I Guerra Mundial supuso un impulso al consumo de tabaco, ya que a los soldados se le entregaba entre sus provisiones. En 1931 aparecieron los cigarrillos con filtro, que rápidamente aumentaron sus ventas (en parte porque a partir de los cincuenta se consideró que disminuían los peligros de cáncer, unido al tabaco bajo en alquitrán). En 1930, se demostró en Alemania la primera correlación estadística, no de causa-efecto, entre el tabaco y el cáncer. También cabe citar el clásico -y amplio- estudio de Hammond y Horn (1954) que relacionaba el fumar con el cáncer de pulmón de tipo bronquiolar, aunque fue muy criticado y no se consideró válido.

En 1964, el Cirujano General de los Estados Unidos, la máxima autoridad sanitaria, anunció por primera vez la relación causal entre el tabaco y el cáncer de pulmón, que era once veces más frecuente entre los fumadores, según el Servicio de Sanidad estadounidense. El Informe del U.S. Departament of Health, Education and Welfare se basaba en una revisión de 32 investigaciones ya publicadas que coincidían en asociar el tabaco con casos de cáncer de pulmón, infarto o parálisis, sin que ningún otro estudio se opusiera a estas conclusiones (posteriormente, en 1988, señaló que el tabaco crea adicción). El Comité de Expertos de la Organización Mundial de la Salud (OMS), a la vista de las pruebas consideradas como concluyentes, proclamó en 1979 la relación entre el tabaco y el cáncer de pulmón. En la segunda mitad del s. XX se han publicado unos 70.000 artículos científicos en los que consta la relación entre la mortalidad prematura y el consumo de tabaco. Hay que tener en cuenta que, si bien el tabaco, directa o indirectamente, se relaciona con la aparición y desarrollo de problemas físicos y psíquicos, tales manifestaciones se producen no de una manera inmediata, sino después del paso de los años (en la mayoría de las ocasiones): de ahí que muchos no consideren las existencia del vínculo tabaco-enfermedad.

Hoy en día, está expandido por todo el planeta, favorecido al cultivarse en casi todos los climas, siendo muchos los países productores. El nuestro es uno de los principales consumidores, sobre todo en la población 25-34 años. Según la Oficina Regional de la OMS, ocupa el quinto lugar-compartido con Francia- en el ranking de los países europeos, por delante de Italia y Reino Unido. Los especialistas señalan diversas peculiaridades económicas, políticas y culturales que propiciaron aquí el afianzamiento de su consumo durante los dos últimos tercios del siglo pasado. Ejemplo de ello son, según Villalbí (1995), la falta de recursos económicos -y pobreza- de la población, el Monopolio estatal sobre el tabaco (que delimitaba la actuación gubernamental en este campo), la recuperación tardía de la democracia y la elevada prevalencia del tabaquismo en profesiones clave. Y aunque la situación ha variado en las 
últimas décadas, especialmente en los últimos años, no puede decirse aún que social y políticamente sea el tabaco una prioridad en la agenda. Otro ejemplo, el primer artículo con datos sobre la mortalidad del tabaco que se publico en España es de 1989 (González Enríquez et al.).

En resumen, el tabaco se descubrió hace varios siglos. Los indios lo usaban en diversas formas, a veces con fines ceremoniales o medicinales, hasta que se constituyó en un simple hábito o placer por fumar. Se empezó a consumir en Europa por distintos tipos de minorías sociales, dependiendo de la época o el país y, debido a este consumo minoritario, su impacto sobre la salud de la población fue prácticamente nulo. Pero la epidemia del tabaquismo empezó hace unos ochenta años en los países occidentales, como consecuencia de la producción y la comercialización masiva de cigarrillos manufacturados. Pronto se popularizó por razones principalmente económicas, pues resultaba barato y altamente rentable para los productores, que invirtieron enormes cantidades de dinero en su promoción, a través de agresivas campañas publicitarias, disparándose en los años treinta su consumo (Dirección General de Prevención y Promoción de la Salud, 1994). Al contrario que pasó con su consumo, que se extendió rápidamente, la adquisición de una conciencia social respecto a su problemática ha sido lenta, distinguiéndose tres períodos distintos:

- En los años sesenta se establecen las evidencias científicas de los principales riesgos vinculados a su consumo, con investigaciones independientes en distintos países, avalados por instituciones sanitarias de ámbito internacional.

- A principios de los setenta, el tabaquismo se consideró como un factor importantísimo causante de incapacidad y muerte prematura, por lo que el control de su consumo se calificó como la acción más eficaz que podía desarrollar la medicina preventiva para lograr la mejora de la salud y la prolongación de la vida.

- A mediados de los setenta, se toma conciencia de que los problemas de tabaquismo no son ni exclusiva ni primordialmente médicos, como otros tantos ámbitos de la salud.

\section{LA INCIDENCIA CRECIENTE DEL TABACO}

En una encuesta realizada en 1997 por el Ministerio de Sanidad y Consumo para conocer la percepción que los españoles tienen de ciertos temas referentes a la salud, un tercio de los encuestados manifestó que fumaban diariamente $(33,1 \%)$. Ya en 1992 , este Ministerio señalaba que el $35,9 \%$ fumaba ( $48 \%$ de los hombres y $25 \%$ de las mujeres), el $51,5 \%$ no y el $12,6 \%$ eran exfumadores. La edad media de inicio del consumo se situaba a los 17 años, sobre todo en la horquilla 12-22 años. 
Sin embargo, otros estudios elevan el porcentaje de la población fumadora en algunos puntos más, con mayor incidencia en el sector masculino, aunque disminuyendo éstos y aumentando las mujeres fumadoras. Entre diez y quince millones de españoles fuman. La última Encuesta Nacional de Salud, con datos de 1997, dados a conocer en el Senado a finales de Febrero de 1999 , recoge que el $35,7 \%$ de la población española mayor de 16 años fuma ( $29 \%$ en los Estados Unidos). No sólo la media de fumadores es más alta que la media europea, sino que la tolerancia es mayor.

La Fundación de Estudios de Economía Aplicada (FEDEA) comparó los datos de las Encuestas Nacionales de Salud de 1987 y 1997. Durante esa década, un periodo marcado por la aparición de acciones y hechos que dejaban constancia de la peligrosidad del tabaco, el porcentaje total de fumadores sólo bajo en un 1\%. Los autores del estudio señalan algunos de los factores que explican la escasa variabilidad: fuerte adicción a la nicotina, falta de éxito de las campañas preventivas y de las restricciones, y la feminización del tabaquismo. Este último dato se comprueba con los cambios en los porcentajes de hombres y mujeres: los primeros bajaron del $54 \%$ al $45 \%$ en esos diez años (la segunda más alta de la Unión Europea), mientras que las fumadoras pasaron del $22 \%$ al $27 \%$. La tendencia coincide con el «Estudio de los estilos de vida de la población adulta española» (Ministerio de Sanidad y Consumo, 1992): la prevalencia de fumadores en España descendió del $40 \%$ de 1978 al $36 \%$ en 1992, disminuyendo el porcentaje de varones fumadores del $65 \%$ al $45 \%$ y aumentando del $17 \%$ al $25 \%$ en las mujeres.

Otras aportaciones de la investigación de FEDEA: en el caso de los varones, el tramo de edad de 30-45 años no ha variado su media de afectados, los más jóvenes se muestran algo más resistentes a empezar a fumar y aumentan los que lo dejan en mayores de 60 años (sobre todo con estudios medios o altos). En las mujeres, son las de mayor nivel educativo las que inflan el porcentaje, especialmente las universitarias que empiezan a fumar. Estos autores señalan que una vez que los sujetos alcanzan los 20 años sin haber fumado, la probabilidad de convertirse en fumador es insignificante. Una prueba más de la importancia del ámbito educativo -y escolar- para prevenir el hábito tabáquico.

Como hemos dicho, casi la mitad de los hombres fuman -los andaluces los que más-, aunque se observa un progresivo aumento de las mujeres jóvenes que se incorporan a este ámbito, tal como hemos indicado. En Andalucía fuma el $38 \%$ de la población, de la que el $32 \%$ lo hace a diario y el resto de forma ocasional, con 1.650 .000 fumadores diarios y otros 350.000 ocasionales. Con respecto a 1994, había 122.000 menos. «El proceso de desarrollo económico y de amplia urbanización habido en los setenta y ochenta comportaron una serie de intensos cambios sociales y culturales. De ellos, la incorporación masiva de la mujer al trabajo y a la educación superior y una mayor libertad de éstas y de los jóvenes en el seno de la moderna familia nuclear propiciaron un mayor uso de esta droga -que no era tenida como 
tal- entre segmentos sociales muy diversos. Una general aceptación social y su escasa incidencia en la modificación de la conducta ha hecho que su consumo no se haya problematizado más allá del daño personal que pudiera causar en la salud de cada fumador. Todo esto unido a los fuertes intereses económicos, incluido el fiscal, ha producido una notable tolerancia en el consumo del tabaco» (EDIS, 1997:37).

Por cierto, un estudio de 1996 del Centro de Investigaciones Sociológicas indicaba que los españoles no fumadores son cada vez más tolerantes con quienes fuman, comparando los datos con otra encuesta similar de 1988. Si bien aumentaban los que opinaban que fumar era perjudicial, mostraban actitudes tolerantes hacia los fumadores, aunque la mitad de los no fumadores se mostraron partidarios de prohibirlo en lugares públicos (y una décima parte que también debería estarlo en privado). El tabaco afecta a los fumadores y a los no fumadores. Los fumadores pasivos inhalan el humo del tabaco de manera involuntaria, estando expuestos a los mismos procesos patológicos que los fumadores activos. Este humo (o "corriente secundaria», frente a la "corriente principal» que aspira el que fuma), tiene una alta concentración de nicotina y monóxido de carbono, siendo tres veces mayor en un cigarrillo encendido que la que contiene la "calada» de un fumador. Además de estas sustancias nocivas, el humo expulsado lleva consigo otras posibles sustancias que están en las vísceras del fumador (en los pulmones, bronquios o estómago).

Una hora expuesto al humo de tabaco equivale a fumar dos o tres cigarrillos, aunque depende de muchos factores ambientales (número de fumadores, tiempo de exposición, tamaño del habitáculo, nivel de tabaquismo, etc.). Según la Asociación Española Contra el Cáncer, estar durante cuatro horas en un espacio con aire contaminado por humo de tabaco hace que un no fumador tenga en la orina y la saliva las mismas cantidades de nicotina que tendría un fumador de tabaco light. Hay que recordar que se han identificado 4.722 sustancias distintas en el humo del tabaco, la mayoría de ellas tóxicas: nicotina, monóxido de carbono (gas tóxico que se une a la hemoglobina de la sangre), alquitrán, sustancias oxidantes... y más de 60 productos cancerígenos (cianuro, DDT, butano). Curiosamente, entre el 40 y $44 \%$ de la población no cree que exista el tabaquismo pasivo.

La revista estadounidense Journal of National Cancer Institute, en el número correspondiente a octubre de 1998, recoge los resultados de una investigación realizada entre 1991 y 1995 con una muestra de más de 2.000 personas, de los que 650 eran no fumadores afectados de cáncer de pulmón. Concluye que convivir con un fumador aumenta un $16 \%$ el riesgo de sufrir esta enfermedad, y un 17\% trabajar -o realizar otra actividad- en un ambiente contaminado por humo. Éstos y otros datos justifican el primer artículo del código europeo contra el cáncer, que en 1987 formuló el Comité de Expertos en Cáncer de la Comisión Europea, con diez recomendaciones para prevenir este mal: No fume. Fumadores, dejen de fumar lo antes posible; pero si fuman, no lo hagan en presencia de otros. 
Según la OMS, en España fallecen entre 2.500 y 3.000 personas no fumadoras por el tabaquismo pasivo (otras fuentes la elevan a 5.000). María Sainz, presidenta de la Asociación de Educación para la Salud (ADEPS), considera la necesidad de "estimular la defensa de respirar sin aire viciado. Provocar que cambie la imagen del fumador. El que fume, que lo haga donde haya fumadores. Hay que combatir el proselitismo silencioso de los fumadores, sobre todo en los lugares públicos, sanitarios y/o escolares. Que tengan su cuarto de fumador, pero que no alardeen de su hábito ante personas no fumadoras e incluso ante los que intenten dejarlo» (1997:76-77). En su opinión, a las personas hay que hacerles partícipes de esta necesidad de una forma activa, abordando cuestiones como:

- El origen del tabaco, su fabricación, empaquetado y venta.

- La incidencia en su medio familiar y comunitario.

- Los perjuicios físicos: catarros, cansancio al mínimo ejercicio, agotamiento intelectual por disminución del oxígeno en el cerebro...

- Los perjuicios económicos: quien gasta diariamente dinero en tabaco, podría tener una partida muy interesante cada mes para otros gastos en cultura u ocio.

- Los perjuicios sociales: se induce a otros a consumir por defender estereotipos de ser "más hombre», "estar a la moda", "la mujer es igual que el hombre», etc. Estos estereotipos son muy rentables a las multinacionales del sector y a las marcas comerciales de otros productos a los que, inevitablemente, se les asocia: café y cigarrillo, coche y cigarro, mujer bonita y cigarrillo, hombre conquistador...

- Las enfermedades asociadas al tabaco.

\section{EL TABACO ¿UNA DROGA?}

Si bien años atrás desde diversos sectores se mantenía que no era una droga, sino un hábito, actualmente los diferentes datos hacen coincidir en considerarlo como tal. "Está claro que el consumo de drogas se ha convertido en un gran problema a nivel mundial. Quizás paliar el de tabaco sea aún más problemático, dado que tanto en los medios de comunicación como a nivel popular se tiende, por lo general, a no incluirlo en el conjunto de las drogas, y a considerarlo como algo habitual, y, en ocasiones, incluso como algo positivo" (Llorent, 1990:38). Es más, a mediados de 1997, los máximos ejecutivos de Philip Morris y Reynolds, empresas líderes del sector, reconocieron en declaraciones juradas que podía provocar cáncer y ser mortal. Ha sido la primera vez que la industria tabaquera admite este hecho, eso sí, obligados por una de las condiciones para el acuerdo a nivel federal para evitar posibles demandas judiciales. 
Sin duda, «la sociedad tiene también una forma concreta de percibir la problemática de las drogas que cambia con el tiempo, lo que no deja de afectar a los comportamientos de los individuos en todo lo relacionado con este asunto. Así, el tabaco ha pasado de ser una droga de inserción a una droga de exclusión" (Vega Fuente, 1993:97). El tabaco es un medio utilizado por el fumador para tomar nicotina, autoadministrándosela para tener el beneficio de sus efectos o para evitar la desagradable sintomatología de su privación. Por lo tanto, es una dependencia, una droga, sea legal o no, aceptada socialmente o no. De hecho, la OMS incluye la dependencia a la nicotina en su última codificación de enfermedades (ICD 9).

La nicotina tiene una capacidad de crear adición tres veces superior a la heroína e inhalada tarda 19 segundos en llegar al cerebro (el "placer» es prácticamente inmediato). La dependencia física y psicológica, la tolerancia que crea su consumo, el síndrome de abstinencia al abandonarlo, la incidencia de la nicotina en el sistema nervioso central y periférico... certifican la naturaleza de droga del tabaco.

\section{EL ENTORNO ECONÓMICO DEL TABACO}

Dediquemos un apartado a conocer la magnitud de las cifras económicas relacionadas con el tabaco: los costes como consecuencia de su consumo, la fiscalidad y los impuestos, las subvenciones para su producción agrícola, precio de venta al público (el Banco Mundial señala que si se eleva un 10\%, el consumo cae un 4-5\%, especialmente entre niños y adolescentes) etc., que sin duda nos ayudarán a comprender una realidad tan compleja. Y es que los Estados se llevan un buen pellizco del dinero que se paga al comprar tabaco, aporta grandes beneficios fiscales a través de los impuestos incluidos en su precio y se incluye en las estadísticas sobre gastos de alimentación dentro de las industrias agrarias, estando protegido por una gran parte de los Gobiernos.

A pesar de las megacifras, no puede argumentarse que el tabaco -y todo el proceso que conlleva- sea un "negocio" rentable, ya que un estudio del Banco Mundial (realizado en Marzo de 1993) concluía que los beneficios económicos que aporta su producción representan sólo la mitad de las pérdidas que produce su consumo, estimadas en $\mathbf{2 0 0 . 0 0 0}$ millones de dólares al año (gastos médicos, bajas laborales, etc.). Asimismo, Peter Anderson, responsable de la Oficina de Tabaco y Drogas de la OMS -con motivo de la II Conferencia Europea de Tabaco o Salud que se celebró en Las Palmas de Gran Canaria en febrero de 1999 (De Vries et al., 1999)- cifró en 30 billones de pts. anuales el coste derivado del tabaquismo para todas las naciones del mundo, descontados los ingresos provenientes de los impuestos que lo gravan.

En España, según un reciente informe del Centro de Investigación de Economía y Salud y el Departamento de Economía y Empresa de la Univer- 
sidad Pompeu Fabra (encargado por el Ministerio de Sanidad y Consumo) el coste anual derivado de las enfermedades asociadas al tabaquismo se sitúa entre los 2.600 y los 3.640 millones de euros (entre 430.000 y 604.597 millones de pts.). Y sólo teniendo en cuenta aquellas dolencias en las que existe un consenso claro en el mundo científico sobre su relación directa con el tabaco, concretamente, el asma, ataque cerebrovascular, bajo peso al nacer, cáncer de pulmón, enfermedades cardiovasculares y enfermedad pulmonar obstructiva crónica, pues no se contemplaron otras enfermedades relacionadas (cáncer de laringe), $\mathrm{u}$ otros costes, como el gasto social indirecto derivado de las bajas laborales, que elevarían las cifras por encima de los 6.000 millones de euros (más de un billón de pts.), semejante a la recaudación fiscal por consumo de tabaco, pero sin contar otros gastos, como la investigación en esas enfermedades. En el 2000 se recaudaron unos 5.430 millones de euros (más de 903.000 millones de pts.) en concepto de impuestos del tabaco.

Hablando de la fiscalidad, la Comisión Europea pretende homologar estos impuestos, exigiendo como mínimo el $57 \%$ de la carga impositiva sobre el precio final y de al menos 70 euros por cada 1.000 cigarrillos $(232,9$ pts. por cajetilla), lo que supondría un total de 299,5 pts. por paquete (138 más que en la actualidad). El desglose de impuesto de un paquete de tabaco en España es el siguiente: Impuesto Especial Ad Valorem (impuesto proporcional sobre el Precio de Venta al Público, del 54\%), Impuesto Especial Específico (es fijo por número de unidades, determinado en la Unión Europea por cada cigarrillo, y en España es de 10 pts. por cajetilla) y el IVA (13,79\% sobre el Precio de Venta al Público). En el caso español, el impuesto grava el $71,36 \%$ del total, pero no llega a lo que propone la Comisión Europea.

La disparidad entre los distintos países es amplia, pues el precio de venta antes de impuestos es muy diferente en cada uno de ellos, por lo que la presión fiscal real también difiere. Aquí, con el impuesto especial y el IVA, la carga es del $57,7 \%$, una de las más bajas de la Unión Europea junto a Italia, Grecia, Irlanda, Portugal y Luxemburgo (en Gran Bretaña es del 64,8\%, 701 pts. por cajetilla en impuestos), con una carga fiscal media de 161 pts. por cajetilla (mientras que la media europea actual es de unas $300 \mathrm{pts}$.). Con la equiparación europea, el Estado recaudaría 232,9 pts. de tasa mínima.

La propuesta de la Comisión -que debe ser ratificada por los Quince por unanimidad-pretende que la fiscalidad del tabaco sea lineal -y no proporcional, como hasta ahora-, la misma en todas la marcas, para que no haya un tabaco o una marca más barata que otra, que haría que los fumadores siempre tuvieran una opción más barata, e incluye otros cambios en cuanto al aumento del tipo mínimo que grava la picadura de tabaco o la modificación de la definición de los puros y cigarrillos (para evitar confusiones entre algunos tipos y los mecanismos evasivos).

Lógicamente, las tabaqueras están en desacuerdo argumentando que una fiscalidad minima por cajetilla, sin tener en cuenta el nivel de vida de los dis- 
tintos países, podría provocar que los Estados con mayor fiscalidad y menor potencialidad se vieran penalizados (y viceversa) y se castigaría a las marcas líderes del mercado. Se quejan de que el tabaco sea el producto más gravado y de que los impuestos sobre cigarrillos han aumentado el $35 \%$ por encima de la inflación, aportando otros aspectos "positivos": el tabaco genera 3 billones de pts. anuales (el casi billón de pts. en impuestos que recibe el Estado, otro 1,6 billones de ventas en estancos y el resto, los beneficios de las industrias tabaqueras) o los 140.000 empleos que dependen de éstas.

También se plantea la exclusión del tabaco como uno de los productos de referencia para el f́ndice de Precios de Consumo (IPC) armonizado europeo, lo cual permitiría a los Estados de la Unión fijar el precio del tabaco sin que "afectara» a los estadísticos económicos, posibilitando medidas disuasorias para combatir su consumo. Según el Instituto Nacional de Estadística, el IPC armonizado que rige en la Unión Europea coincide, prácticamente, con el nacional, referencia como sabemos para las subidas salariales, pensiones, etc. No es baladí la relación tabaco-IPC. Los españoles dedican 2,3 de cada 100 pts. a la compra de tabaco (la misma proporción, por ejemplo, que a la adquisición de ropa). A la vista de esto, podríamos encontrarnos con un IPC irreal si no lo contempla. Según el último informe del Observatorio Español sobre drogas (dado a conocer en junio de 2001), gastamos más de dos billones de pts. al año en tabaco y alcohol, las dos drogas de mayor penetración social, casi la mitad que el presupuesto anual del Ministerio de Sanidad (1.255.387 pts. al año al tabaco por persona de media). Resulta paradójico que distintos datos señalen que el gasto anual medio para consumo de tabaco por hogar en muchas familias españolas es superior al gasto destinado para educación (Solano Reina, 1997).

La venta de cigarrillos (que supone el $94,6 \%$ del tabaco) se mantiene estable, en torno a los 4.345 millones de cajetillas al año. En el transcurso de una década, de 1989 a 1999, creció en 8,6 paquetes por habitante (de 145,5 cajetillas de entonces a 159,3). Sólo descendió un 0,2\% en Castilla y León. Por contra, el gasto en alcohol decreció un $0,72 \%$, sobre todo en los que beben a diario, pero aumentando los que lo hacen ocasionalmente. En el 2000 se vendieron 3.385 millones de paquetes, de los que 1.040 fueron de tabaco negro (disminuyó sus ventas un 1,3\%, mientras el rubio aumentó un $7,5 \%)$ y 586 millones de rubio light. Según datos recogidos en la revista $E l$ Fumador -editada por el Club de Fumadores por la Tolerancia-, durante el primer semestre de 2001 se vendieron 2.191 millones de cajetillas (110 millones más que en el mismo periodo del año anterior), obteniendo el grupo Altadis - resultado de la fusión entre Tabacalera y la francesa Seita, que tiene actividad en tres áreas: cigarrillos, cigarros y logística- un beneficio neto de 29.367 millones de pts. (176,5 millones de euros), un $34,3 \%$ más que en el mismo periodo de tiempo del año anterior, y el beneficio bruto de explotación aumentó un 13\% (71.046 millones de pts.-427 millones de euros), que en el segundo semestre se esperaba situar entre el 15 y $20 \%$. Según Altadis, su cotización de Bolsa en el 2.000 se vio penalizada por fac- 
tores externos sociales, ya que el mercado estaba muy sensible ante las informaciones que se produjeron (demandas en los Estados Unidos, nuevas Normativas, posible armonización fiscal). Pero consideran que eran causas localizadas que no deben afectar una vez pasadas.

Más números: la X Conferencia Mundial Tabaco o Salud celebrada en Pekín aportó datos preocupantes. China es el país con más fumadores: el $70 \%$ de los varones adultos, el $10 \%$ de las mujeres y el $40 \%$ de los menores de edad de una población de 1.200 millones de habitantes. Es el mayor productor, con casi dos billones de cigarrillos al año (cuatro veces más que el segundo, los Estados Unidos). Sin embargo, Grecia es el mayor consumidor por habitante: casi 140 paquetes de cigarrillos anuales. La producción mundial anual es de unos 1.000 cigarros por persona. El Informe del Programa de Naciones Unidas para el Desarrollo 1998 (PNUD) señala que en Europa gastamos 50.000 millones de dólares en tabaco. En 1996, la venta de cigarrillos llegó a los 45 billones de pts. y se habla de unos beneficios de 3 billones de pts. Sólo la Philip Morris tiene unos beneficios de 6.300 millones de dólares (dedicando unos 600 para atender indemnizaciones). Un dato paradójico: en 1995 la Comisión Europea dedicó 2.500 millones de pts. a campañas «antitabaco", frente a los 160.000 millones que destinó a subvencionar los cultivos de tabaco. Nos encontramos así con otra paradoja: las ayudas al cultivo.

El informe «Estrategia de desarrollo sostenible» de la Presidencia de la Comisión Europea, que tiene como objetivo adoptar iniciativas para lograr un mejor nivel de calidad de vida para el futuro, plantea la necesidad de eliminar en breve plazo las ayudas comunitarias y estatales en diversos sectores, como el carbón (un billón de pts. anuales) y el tabaco (151.000 millones de pts.). España recibió 18.500 millones de pts. como ayudas en el 2000 (otras fuentes consultadas las cifran en 13.000, alejada en todo caso de los 62.000 que ingresó Grecia o los 58.000 millones en Italia). Uno de los argumentos en que se basa esta propuesta es la contradicción existente en los propios Estados, que dedican cantidades para disminuir el consumo de tabaco, mientras financia o subvenciona su producción. A ello hay que añadir que diversos organismos públicos plantean presentar demandas judiciales contra las tabaqueras por los perjuicios sanitarios. Así, los Quince han apuntado la intención de suprimir las subvenciones al tabaco, a pesar de que en Europa se produce el $23 \%$ de lo que consume. El sector agrícola afecta a 200.000 personas en la Unión Europea, fundamentalmente pequeñas explotaciones. En España, del tabaco dependen 6.500 cultivadores, genera unos tres millones de jornales al año y afecta -directa o indirectamente- a unas 30.000 familias. Según la Compañía Española de Tabaco en Rama, tenemos una producción anual de 42.300 toneladas, el $13 \%$ de la producción de tabaco de la Unión Europea. Supondrá un problema muy focalizado en algunas áreas: de las 14.281 hectáreas dedicadas a su cultivo, unas 12.000 están en Cáceres, produciendo el $88 \%$ de la cosecha nacional. El resto, 1.500 has. están en Granada, 350 en León y Ávila, 150 en Toledo, 80 en Navarra y 12 en el País Vasco. 
Y las subvenciones económicas son fundamentales, pues de los ingresos que genera un kg. de tabaco en España, sólo una sexta parte deviene de su precio de venta y el resto corresponde a ayudas comunitarias. Según los números de la Mesa para la Defensa del Sector Tabaquero, el importe de la cosecha del 2000 fue de 22.277 millones de pts., de los que 18.591 fueron por primas a los cultivadores (el 84\%) y 3.686 a la venta comercial del producto. De la misma forma, en Mayo de 2001, la comisión presidencial de los Estados Unidos informó que daría unos 2 billones de pts. a los agricultores que reduzcan o dejen la producción de tabaco, acabando con el antiguo sistema de cuotas que durante décadas ha sustentado a la industria tabaquera. Allí -con 50 millones de adultos fumadores, que dan lugar a 430.000 personas fallecidas al año por enfermedades relacionadas con el tabaco- se recaudan unos tres billones por impuestos (el $35 \%$ del precio de venta corresponde a este concepto). Japón ingresa unos cuatro billones de pts.

El consumo de tabaco se ha incrementado en más del $150 \%$ en los últimos 30 años, mientras que el aumento de población sólo ha sido del $22 \%$. El consumo per cápita de cigarrillos se redujo en un $10 \%$ entre comienzo del decenio de 1970 y principio de los noventa en los países industrializados, pero aumentó un $64 \%$ en los países en vías de desarrollo (se duplicó con creces en Haití, Indonesia, Senegal, Siria, etc. o se triplicó en Camerún o Nepal), cifrándose el incremento anual de fumadores en los países del Sudeste Asiático un $5 \%$ y en Sudamérica un $2 \%$ (las compañías tabaqueras recogen en sus informes la importancia de países de África y Europa del Este como grandes mercados potenciales a corto y medio plazo, sobre todo por la relación entre tabaco-cultura occidental). En 1971, el consumo en China era de 730 cigarrillos por adulto y año, y veinte años más tarde ascendía a 1.900 cigarrillos. Es el ejemplo más claro del incremento que se da en los países de rentas bajamedia (pasaron de 860 a 1.410) mientras que los más desarrollados bajaron de 2.860 a 2.590 cigarrillos. $Y$ es que las tabaqueras necesitan reclutar diariamente a unos 8.000 nuevos fumadores para paliar los que dejan de fumar (muchos por muerte) para mantener el nivel de ganancias. Y se buscan en nuevas "plazas": países del Este, del Tercer Mundo... o en adolescentes y mujeres de los mercados "occidentales», objetivo específico de la industria tabaquera (Iranda, Silero y Andradas, 2000).

\section{MEDIOS DE COMUNICACIÓN SOCIAL Y TABACO}

Los mass-media están omnipresentes en la sociedad actual, de tal forma que constituyen una de las características más definitorias de la misma. La educación para la salud también se ve afectada por la incidencia de los medios de comunicación social. El tabaco, su consumo y representación es uno de los aspectos donde más claramente se explícita esta relación. ¿Tan importante es la publicidad, el papel de los medios en la promoción del taba$c o$ ? Veamos un ejemplo analizado por muchos expertos y observadores que resulta ciertamente significativo. En 1994, las estadísticas señalaban que en 
los Estados Unidos el $22,6 \%$ eran adultos fumadores, continuando con la bajada que comenzó en los ochenta (en 1993 era mayor del 25\%). Ahora supera el 30\%. Algunos explican este hecho señalando como una de las culpables a la industria del cine, que sitúan como modelos a seguir a actores que fuman en escena, teniendo en cuenta que, del millón y medio de nuevos fumadores que surgen cada año, un millón son menores de edad. Así, en 1994, muchos actores aparecían en escena fumando en películas de éxito comercial. Uno de los más señalados fue el "renacido" John Travolta (que en verdad no es fumador) en Pulp fiction. El aumento de actores y actrices fumando en los films se ha afianzado desde principios de los noventa, cuando en años anteriores la tendencia era contraria: en los setenta sólo lo hacía el $25 \%$, en 1994 la mitad y hoy casi el $80 \%$ de las películas muestran a un actor fumando.

En 1992, ante la prohibición de la publicidad del tabaco en la Comunidad Europea, dos significativas personas -ya que tiempo atrás habían trabajado como modelos publicitarios para la industria tabaquera- escribieron una carta apoyando tal iniciativa: Dave Goerltz, que apareció en 42 anuncios de Winston, que por entonces tenía problemas de salud relacionados con el tabaco, y Wayne McLaren (con Marlboro) que murió meses más tarde por un cáncer de pulmón. En la misma línea se situó la modelo Jane Sackman (Lucky Strike), que sufrió un cáncer de laringe por el que fue laringectomizada.

Otro dato: la Comisión Federal de Comercio de los Estados Unidos presentó una denuncia judicial contra la campaña publicitaria de Camel, que utilizaba un sonriente camello tipo "peluche», que muchos asemejaban a un personaje de dibujos animados. A raíz de la campaña de anuncios, aumentó un 13\% el consumo de dicha marca en niños, jóvenes y/o adolescentes. En diciembre de 1998, la Asociación Española de Tabaco -que agrupa al 95\% de los fabricantes que operan en nuestro país- ratificó la "Primera Declaración de Principios y Compromisos Básicos en relación con la Publicidad y la Prevención del Acceso de los Menores al Tabaco", conjuntamente con la tercera edición del Código de Autorregulación de la Publicidad sobre el mismo. De esta Declaración y Código, para evitar el consumo entre los menores, se deduce que las empresas tabaqueras españolas defienden su derecho a comercializar un producto legal, aunque evitarán que su publicidad llegue a los más pequeños. Por ello, en las campañas publicitarias no se empleará a modelos famosos, ni actores menores de 25 años, ni aparentarán tener menos de dicha edad. Tampoco se hará campaña a menos de 200 metros de colegios con menores, ni en las sesiones de cine para menores de dieciocho años, etc.

Por otro lado, las campañas informativas sobre el daño que produce el tabaco han supuesto generalmente una disminución en su consumo, aunque al tiempo de cesar aquéllas, se volvía a incrementar. Hay que recordar que «el tabaco (y el alcohol) son sustancias que han pasado de ser la expresión de un modo de vida, sobre todo en la cultura mediterránea, a convertirse en 
pasaporte hacia la evasión. Sobre todo para el adolescente, codiciado sujeto/objeto de la publicidad. Es por tanto a las mujeres en general y al grupo de edad adolescente donde se dirige la mirada, el esfuerzo y los millones que las compañías tabaqueras invierten en la publicidad que les garantice el abastecimiento de muchos consumidores, ya que entre el resto de la población, en el caso de los fumadores, la tendencia es a disminuir el consumo y, en muchos millones de personas ya, el abandono del hábito» (Dirección General de Prevención y Promoción de la Salud, 1994:22).

\section{EL TABACO Y LOS JÓVENES}

Los medios de comunicación ayudan a que en la adolescencia se pruebe por primera vez (debido a la falta de una personalidad definida, carácter inmaduro, por curiosidad, deseo de nuevas experiencias, por efecto de la imitación al tomar como ejemplo a los adultos, ser igual a otros del grupo de iguales). La edad de inicio es cada vez más temprana (la mitad de los fumadores empiezan antes de los 14 años). Algunos estudios indican que más del 95\% se iniciaron en la adolescencia, manteniéndose posteriormente debido al hábito, la costumbre y la acción de la nicotina. Es llamativo e importante para abordar convenientemente el problema el dato de que el $90 \%$ manifiesta que la primera vez que fumó lo hizo en compañía de otros jóvenes (sólo un $10 \%$ en solitario).

Bajo la coordinación de la OMS, periódicamente se lleva a cabo, de manera simultánea en distintos países europeos, un estudio con 12.000 alumnos de entre 11 y 18 años sobre las conductas de los escolares relacionadas con la salud. En España, corre a cargo de un Grupo de Investigación con miembros de la Universidad de Huelva y de la Universidad Ramón Llull de Barcelona. Merece la pena analizar un cambio significativo entre los datos de 1990 y los de 1994. A principios de los noventa tuvo lugar un apreciable descenso en los jóvenes (en 1987 el 48,2\% de los 16-24 años se declararon no fumadores, que aumentó al $56,7 \%$ en 1993), para posteriormente tender a aumentar, sobre todo en las chicas y a partir de los 15 años, en los que se observa un empeoramiento en los hábitos de salud. Otros datos de interés de la encuesta de 1994: el 49\% de los jóvenes han confirmado haber fumado (la mitad lo hacen regularmente); por primera vez, las chicas superan a los varones en la adicción; se observa una estabilización en el consumo a los 11 años, descenso en los varones y aumento en las jóvenes de 13 años, mientras que a los 15 el porcentaje de chicos consumidores está estabilizado con respecto a estudios anteriores, frente a un incremento de mujeres.

Otros estudios señalan que el tabaco ha sido probado por el $70 \%$ de los estudiantes de Bachiller (de los que el 30\% fuma a diario) y el $45 \%$ de los de Primaria-EGB (un $8 \%$ lo hace frecuentemente), siendo mayoría las mujeres, aunque los grandes consumidores son casi siempre chicos (Torres, 1990). La OMS señala que España es uno de los países donde más precozmente se 
contacta con el tabaco (Barrueco Ferrero, 1998). Existen al menos cinco factores que explican por qué los jóvenes -ampliable a la población en generalconsumen tabaco (Mendoza, Villarrasa y Ferrer, 1986:203-204):

- Es una droga que se anuncia.

- Las grandes empresas de tabaco presionan eficazmente a los Gobiernos.

- La población no recibe una información veraz sobre el tabaco.

- Es una droga barata y se vende en todas partes.

- Muchas personas que deberían ser modelos a seguir fuman.

Es más, para los jóvenes, el tabaco tiene un gran significado simbólico, estando muy relacionado con las comunicaciones sociales. Es uno de los mediadores para entablar comunicación. Ya en el 1977, C. R. Jesser y S. Jesser señalaban que las actitudes y normas del grupo de iguales varían en relación al consumo de tabaco (en general de las drogas) antes de que los usuarios empiecen a consumirlos. Este cambio de actitudes -y posteriormente de las conductas- se debe a la incidencia de varios factores, de los cuales cada sujeto selecciona y asimila las vivencias incorporándolas a su caso concreto: a) la observación de la conducta de los padres al fumar; b) la interpretación de los padres sobre el consumo de estas sustancias; c) y la observación del consumo entre el grupo de iguales y de los valores religiosos.

\section{DENUNCIAS QUE PUEDEN CAMBIAR EL ESCENARIO}

Años atrás, en verano de 1997, con motivo del Día Mundial sin Tabaco -la OMS dio el lema de "Todos unidos por un mundo sin tabaco»-saltó a la opinión pública un debate sobre el tabaco. A raíz de las importantes demandas judiciales contra las compañías tabaqueras estadounidenses, entre otros motivos, los medios de comunicación se hicieron eco de noticias y reportajes sobre el tabaco, su consumo y consecuencias. En los últimos años se han presentado numerosas denuncias relacionadas con su consumo y, aunque pudiera parecer que se escapan del ámbito educativo, no está de más comentar brevemente la situación actual, por cuanto son hechos que tienen una repercusión muy directa en el tema.

Desde los Estados Unidos, conocimos la propuesta de acuerdo a nivel federal para que la industria tabaquera pagase 57 billones de pts. para afrontar los gastos sanitarios de los fumadores a cambio de que éstos no demandaran individualmente a aquéllas (aunque hubo opiniones contrarias al considerar que supondría la inmunidad "de por vida» de los productores). En noviembre de 1998 se llegó a un acuerdo entre las tabaqueras estadouniden- 
ses y 48 de sus Estados por el que aquéllas aportan 200.000 millones de dólares a lo largo de 25 años a cambio de eludir posibles juicios. Llamó sin duda la atención los aproximadamente catorce millones por hora que cobraron como minuta los abogados de los Estados implicados.

Sin duda, los recursos dedicados en este sentido pueden determinar el éxito de las acciones. El ex-presidente estadounidense Bill Clinton intentó que la agencia estatal que regula los alimentos y medicamentos (FDA) reglamentara el comercio del tabaco, como principal instrumento de control gubernamental, partiendo de que la misma puede incidir en cualquier producto que afecte a la estructura o función del cuerpo humano. Sin embargo, en marzo de 2000 el Tribunal Supremo estableció que la FDA carece de jurisdicción para regular los productos del tabaco y sentenció que no es una droga susceptible de ser controlada como tal, devolviendo al Congreso la autoridad para su posible control, donde los fabricantes de tabaco tienen notables influencias (quizás la industria tabaquera sea el ejemplo por excelencia de cómo actúa un lobby de presión política). Las restricciones de venta planteadas por Clinton se centraban en diversos ámbitos, tales como obligar a los establecimientos a identificar a cualquier persona que aparentara no ser mayor de edad y limitar las máquinas expendedoras a los lugares en los que sólo puedan acceder los adultos.

A raíz de la elección de George W. Bush como presidente, el Departamento de Justicia puede abandonar la demanda federal que Clinton ordenó presentar contra las tabaqueras (similar a la que presentaron conjuntamente numerosos Estados y que se cerró en 1998 por un acuerdo extrajudicial), pues aquél ha recortado los fondos destinados a ello: los abogados de la demanda calcularon que necesitaban 11.000 millones de pts. y lo aprobado finalmente han sido sólo 350 millones de pts., cuando el Departamento de Justicia lleva recopilados más de 73.000 millones de páginas de documentos de todo tipo.

Un enfermo terminal de cáncer de 56 años consiguió que un juez de Los Ángeles condenara a Philip Morris a una indemnización de ii586.000 millones de pts.!! (más de una cuarta parte de los beneficios anuales de la empresa), convirtiéndose en el veredicto dictado con mayor cuantía en favor de una persona en la historia judicial estadounidense y la mayor condena a una tabaquera. La demanda se basaba en que ésta engañaba al fumador, pues no le informó de los riesgos para la salud al consumir tabaco, y el jurado dictaminó el pago de los daños punitivos por fraude, negligencia y por fabricar un producto defectuoso, además de otros 1.000 millones en compensación por los daños causados. Semanas antes, otro jurado estadounidense sentenció a diversas tabacaleras a pagar 5.920 millones de pts. a una aseguradora.

Sin embargo, otras denuncias no se han ganado: una azafata de aviones trabajó entre 1973 y 1996, tuvo que retirarse por motivos de salud y en 1997 se le diagnóstico sarcoidosis, una enfermedad cuya causa se desconoce. Pre- 
sentó una demanda contra los cuatro grandes fabricantes estadounidenses (Philip Morris, R.J. Reynolds, Brown \& Williamson y Lorillard) exigiendo indemnizaciones millonarias por los costes médicos y la pérdida de ingresos, argumentando que el "humo de segunda mano" de las cabinas de las aeronaves afectó a su salud. Actualmente, con 59 años, minusvalía permanente y utilizando oxígeno para poder respirar, está en lista de espera para someterse a un trasplante de pulmón (padece además bronquitis crónica y enfisema). Un jurado de Miami debía decídir si ese humo causó o agravó la enfermedad de la ex-azafata y determinó en abril de 2001 que la industria tabaquera Unidos no era responsable de la enfermedad pulmonar que padecía. Es un ejemplo de otros muchos casos de demandas que los sobrecargos y azafatas han presentado, argumentando que han sufrido enfermedades debido al humo de cigarrillo presente en los aviones cuando en los vuelos de los Estados Unidos todavía no se había prohibido fumar.

En Europa, la línea argumental de las resoluciones judiciales no coincide con algunas de las acontecidas en los Estados Unidos favorables a los demandantes. Sólo un caso, en Francia, se resolvió culpando en parte a la industria tabaquera, aduciendo que el demandante había empezado muy pronto a fumar, a los 15 años, y que por el periodo en que no era consciente del peligro de ello, debido a su corta edad, debía ser indemnizado. Otras denuncias han sido institucionales, pero no por motivos de salud. En Agosto de 2001, la Comisión Europea y diez de los quince miembros de la Unión Europea (Alemania, Bélgica, España, Finlandia, Francia, Grecia, Holanda, Italia, Luxemburgo y Portugal) presentaron una nueva denuncia contra Philip Morris y R.J. Reynolds, al considerarlas directamente implicadas en la organización del contrabando de tabaco que llegaba a Europa desde la década de los setenta, continuación de otra presentada en noviembre de 2000 y desestimada en julio de 2001 por un tribunal de Nueva York, al argumentar el juez que el contrabando de cigarrillos no supone un «daño directo» a los recursos financieros de la Unión, por lo que ésta ni los Estados eran competentes para presentar la denuncia a raíz de las pérdidas por los ingresos aduaneros y los impuestos indirectos dejados de percibir, calculados desde Bruselas en 180.000 millones de pts. al año.

En España, la primera sentencia por un caso de tabaquismo fue dictada en abril de 2000, presentada la denuncia por un hombre que había perdido los brazos y las piernas por el síndrome de Buerger, pero el juez consideró no acreditado el nexo causal entre el consumo de tabaco y la enfermedad. Coincidiendo con este argumento, en septiembre de 2000 se absolvió a Altadis en un segundo caso presentado en Palma de Mallorca por otra enferma que también padecía el síndrome de Buerger, reclamando una indemnización de 900.000 euros (150 millones de pts.). La tercera sentencia se dictó en marzo de 2001 y la denuncia fue presentada en Barcelona en enero de 1998 por los familiares de un fallecido en 1993 por cáncer de pulmón, "causado" por el consumo de tres paquetes de cigarrillos diarios (fumaba desde hacía 20 años), que solicitaban una indemnización de 60 millones de pts. El fallo se basa en que no se demos- 
tró una relación directa y exclusiva entre el hábito de fumar y la enfermedad, pues podían ser otros los «factores de riesgo» que la pudieron propiciar. No obstante, es la primera vez que se reconocía la relación contractual entre Altadis y el fumador, y la juez destaca como «hecho notorio y conocido" el peligro del tabaco, pero no se demostró que la causa de la muerte fuera su consumo.

La cuarta sentencia, favorable también a las tabaqueras -en este caso, Altadis y Philip Morris Spain-, fue a raíz de la demanda que presentó la viuda de un médico que murió en 1999 de infarto de miocardio y cardiopatía isquémica, alegando la relación causal entre éstos y el consumo de tabaco. La resolución de un Juzgado madrileño desdice los dos fundamentos de la demanda en cuanto a la causalidad ( no puede afirmarse que el consumo del tabaco fuera la causa fundamental del deterioro de la salud del fallecido") y en cuanto a la falta de información ( «es de general conocimiento" el riesgo del tabaco, siendo el fallecido además médico y pudo dejar de fumar sin uso de ningún tratamiento). Otra demanda presentada en Sueca (Valencia) por un enfermo de bronquitis crónica que pedía una indemnización de cien millones de pts. ha sido también archivada.

En febrero de 2001, un Juzgado de Primera Instancia admitió a trámite la demanda presentada por la Asociación de Laringectomizados (extirpados de laringe) de Barcelona contra la industria tabaquera española (Altadis, Compañía de Tabacos de Canarias y la distribuidora Logista) y estadounidense (Philip Morris, Reynolds y British American) reclamando 3 millones de euros (quinientos millones de pts.) para la compra de un local dotado de los medios suficientes para atender a sus miembros y sufragar los casi treinta millones de pts. anuales que requieren para gastos médicos, logopedas y foniatras para enseñar a hablar a los que han perdido sus cuerdas vocales. Era la primera Asociación -650 afectados por cáncer de laringe- que presentaba una demanda de este tipo contra los fabricantes. Otra demanda fue presentada por la Asociación Leonesa de Laringectomizados, desestimándose en septiembre de 2001 la petición de suspender cautelarmente la fabricación, comercialización y distribución del tabaco en España por «falta de jurisdicción», al igual que la publicación en las cajetillas del benzopireno que contiene cada cigarrillo (recordemos la polémica que produjo este producto en el caso del aceite de orujo, y estas Asociaciones consideran que un paquete contiene tanto benzopireno como nueve litros de aquel). Coincidiendo con lo anterior, también la Asociación de Laringectomizados de Vizcaya (1.015 socios) presentó una demanda que tuvo su vista preliminar en septiembre de 2001.

Las demandas suelen reclamar indemnizaciones a las tabacaleras, pero en algunos casos se han presentado contra otros estamentos. Así, por ejemplo, una ex-fumadora malagueña ha actuado contra la Administración Central al considerar que adquirió el hábito cuando el Estado tenía el monopolio de la importación, producción y venta de tabaco, y solicita que el Estado le reembolse el dinero del tratamiento para dejar de fumar, presentando una demanda ante la Sala de lo Contencioso del Tribunal Superior de Justicia de Anda- 
lucía, que la admitió a trámite a finales del primer semestre del 2000. Pero, considerando que el demandado es el Secretario de Estado de Hacienda, la pasó diez meses después a la Audiencia Nacional para su resolución. La indemnización solicitada es de unos 250 euros (algo más de 40.000 mil pts.), el coste que gastó en una terapia de deshabituación por la que dejó de fumar después de hacerlo durante 25 años, dado que el tratamiento no estaba incluido en las prestaciones públicas. Ha habido otros juicios en el ámbito laboral, o el de un estudiante que dejó sus estudios de la Facultad de Derecho de la Universidad Complutense de Madrid debido a las molestias causadas por el tabaco que fumaban sus compañeros en el aula.

\section{OTROS HECHOS SIGNIFICATIVOS}

Veamos seguidamente otros hechos preocupantes que nos ayudan a conocer la realidad del tabaco. En un comunicado de la OMS con motivo de una reunión preparatoria de las negociaciones sobre la futura Convención sobre el Control del Tabaco, que debería ser adoptada y ratificada en mayo de 2003 (negociaciones que se remontan a 1991, cuando los Estados miembros de la OMS se comprometieron a iniciarlas), considerada por la agencia de Naciones Unidas para la salud como el primer instrumento internacional para evitar la expansión de la producción y consumo de tabaco se señala que «existen cada vez más pruebas que demuestran que la nicotina ha sido utilizada con el fin de que los jóvenes se conviertan en dependientes y sigan siendo fumadores». Las estadísticas de niños fumadores en Brasil, China, Egipto, India, Omán, Sudáfrica, Zimbabue y otros países, corroboran esta preocupación.

Un primer borrador de la Convención propone que la industria tabaquera «se haga responsable del daño que sus productos causan a la salud y el medio ambiente" y pretende consensuar un texto común que todos los países ratifiquen, con «el objetivo final de proteger a las generaciones presentes y futuras de las devastadoras consecuencias sanitarias, sociales, ambientales y económicas que tiene el consumo de tabaco y la exposición a su humo». Ya en mayo de 1999, la Asamblea de la OMS aprobó por unanimidad la resolución titulada "Liberarse del tabaco", antesala del pretendido Convenio Mundial de Lucha contra el Tabaco, para fomentar medidas combinadas en el ámbito internacional para reducir su consumo: prohibición de la publicidad y promoción, aumento regular y constante de los impuestos, mejor acceso a los productos sustitutivos, creación de coaliciones antitabaco...

No obstante, las negociaciones no tuvieron éxito total en la segunda ronda celebrada a principio de mayo de 2001 , por lo que habrá que esperar a lo que ocurra en la tercera a celebrar en diciembre en Génova. En este sentido, la Alianza para la Convención-marco, constituida por 125 organizaciones no gubernamentales de 50 países, calificó como fracaso el encuentro debido a que ciertos países (Estados Unidos, Alemania, México y Japón, especialmente) "antepusieron los negocios a la salud pública». 
Jonathan Klein (Rochester Clinic Hospital de la Universidad de Nueva York) y el abogado Steve St. Clair realizaron un interesante estudio que dieron a conocer en agosto de 2000 en la British Medical Joumal. Analizaron numerosos documentos de las compañías tabaqueras utilizados en diferentes juicios, demostrando la connivencia entre estas empresas y las de dulces que elaboraban los conocidos cigarrillos de chocolate y otros productos con la apariencia de cajetillas de tabaco. Las tabaqueras eran conscientes de niños que jugaban con los cigarros de chocolate y llegaban a adolescentes consumiendo tabaco. Los fabricantes de dulces también ocultaron estos datos y llegaron a manipular sus propios estudios sabedores del papel promocional que suponía imitar las formas o nombres de tabaco ('Pell Mell' por 'Pall Mall' o 'Cool' en vez de 'Kool'). Algunos estudios que encargaron cifraban en el doble la posibilidad de que llegaran a ser fumadores. Significativo fue el realizado en 1991 por el Dr. Howard Kassinove, que de 76 páginas el original, una vez "cocinado" y "limpiado», quedó en 31. No es extraño, pues, que muchos Estados prohíban la venta de estos dulces (Australia, Canadá, Finlandia, Noruega, Reino Unido y numerosos países del Medio Oriente).

Importante revuelo tuvo lugar en julio de 2001 cuando el Wall Street Journal dio a conocer parte del contenido de un informe encargado por Philip Morris - a instancias, más concretamente, de su filial en la República Checaa la consultora estadounidense Arthur D. Little International sobre el tabaco en dicho país. Aseguraba que su consumo podía tener efectos positivos para las «finanzas públicas... por los ahorros en cuidados médicos a causa de una mortalidad prematura». El ahorro para el Gobierno Checo se cifraba, al cambio, en casi dieciocho millones de euros (tres mil millones de pts. aproximadamente). Lógicamente, la tabaquera tuvo que salir de manera inmediata a enmendar la noticia. El vicepresidente de la multinacional calificó el informe de «tremendo error... falta grave... decir que es inapropiado es quedarse corto; es un análisis deplorable y una falta de respeto total e inaceptable hacia los derechos humanos», y a declarar que "nadie se beneficia de las muy reales y serias enfermedades causadas por el tabaco». A raíz de ello se suspendieron estudios similares que se venían realizando en otros países del entorno (Eslovaquia, Eslovenia, Hungría, Polonia, etc.).

Gracias a esos movimientos y recopilaciones de materiales de diversa naturaleza, hemos conocido más y mejor las actuaciones de las tabacaleras. Así, por ejemplo, Philip Morris preparó un grupo de presión en nuestro país a principios de los noventa con el objetivo de dificultar cualquier posible medida antitabaco. Estos materiales se conocieron recientemente en gran medida por el trabajo recopilatorio de diversos profesores estadounidenses de la Escuela de Salud Pública de John Hopkins, que, a su vez, dieron la información al Comité Nacional para la Prevención del Tabaquismo. Cabe destacar en este sentido los informes "Corporate Affaire Work Plan Spain and Portugal" (Plan de Trabajo para España y Portugal, Philip Morris, 1990) y "Social Acceptability Program: Initial Recommendations for Spain" (Programa de Aceptación Social: Primeras recomendaciones para España, realizado por la 
empresa de comunicación Burson-Masteller en 1992). En ellos se informa de la necesidad de influir en científicos, investigadores y personas relevantes en pro del tabaco (como una forma de manifestación de la libertad), elaboración de literatura científica en esta línea, en paralelo a desprestigiar (o restar credibilidad) los artículos contrarios, edición de discos, libros, fomentar encuentros científicos y debates, apoyo a asociaciones de prestigio (las tabaqueras, han confirmado haber pagado a científicos para acudir a reuniones de la OMS)... incluso "presionar a los funcionarios de salud».

\section{ACTUACIONES DESDE DISTINTOS ÁMBITOS}

Navarra fue la primera Comunidad que financió parcialmente terapias para dejar de fumar. Desde 1995, paga un tercio del tratamiento de deshabituación, concretamente los costes de los parches de nicotina del segundo de los tres meses que dura. En 1999, aproximadamente 400 personas se acogieron a la experiencia, en tratamientos individuales o grupales en distintos Centros de Salud, lo que supuso un desembolso medio para la Hacienda Foral de unas 10.000 pts. A partir del 2000, financia el $66 \%$ hasta 20.000 pts. y ello supuso una mayor demanda (en los primeros seis meses se igualó el total del año anterior). A título informativo, los parches transdérmicos se comercializan en tres variedades de envases, dependiendo de la carga de nicotina sustitutiva y tienen un coste cada uno de entre 30 y 36 euros (5.0006.000 pts.). Un fumador de un paquete diario necesita para el primer mes un envase de 14 parches con un total de 52,5 mg. de nicotina. Los 14 parches del envase para el segundo mes suministran $35 \mathrm{mg}$. y en el tercero, 17,5 mg.

En mayo de 2001, la Generalitat catalana comenzó en tres comarcas un programa que costea hasta 50.000 pts. del tratamiento farmacológico de deshabituación correspondiente a las terapias sustitutivas de la nicotina (chicles, parches, aerosoles nasales e inhaladores bucales) y los fármacos complementarios, que usualmente tienen como principio activo el bupropión, antidepresivo que actúa en los centros cerebrales de recompensa mejorando el estado de ánimo, y que tiene un alto índice de eficacia (en torno al $25 \%$, según diversos estudios, porcentaje de personas ex-fumadoras que siguen sin fumar un año después de abandonarlo). Aquella cantidad se reembolsará cuando a los seis meses se compruebe en un control médico que se ha dejado de fumar. Esta campaña se dirige a fumadores habituales mayores de 16 años, que, a través del médico de cabecera-familia (o del farmacéutico), tendrán un seguimiento, por medio de un carnet, de los medicamentos administrados y de la carencia de consumo de tabaco. Los médicos y enfermeros reciben gratuitamente chicles o parches de nicotina.

Por otro lado, en Andalucía se estudian diversas medidas para incentivar el abandono del tabaco, destacando la posibilidad de conceder vacaciones a los profesionales sanitarios y educativos que lo dejen, basándose en el estudio de la Sociedad Andaluza de Medicina Familiar y Comunitaria 
realizado en 101 Centros de Salud, según el cual «si los médicos aconsejasen a sus pacientes que dejasen de fumar, al menos un $10 \%$ lo abandonaría», lo que supondría que unos 100.000 andaluces lo dejarían. Canarias, Castilla y León, Cataluña, Galicia y La Rioja organizan el concurso Déjalo y gana, sorteando premios para los que dejan de fumar (viajes sobre todo). Hay otras experiencias, como Ayuda Psicológica a través de consultas telefónicas.

Otras acciones se enmarcan en el marco judicial. La Junta de Andalucía ha demandado a doce multinacionales, al considerar el tabaquismo como una fuente de enfermedades que llegan precedidas por una publicidad engañosa, en cuanto que no predican sus excelencias, sino la excelencia social de fumar, por lo que las tabaqueras producen un «daño propio y típico de su actividad" que no asumen, sino que lo traspasan a la Sanidad Pública: entre todos pagamos los daños y las empresas se llevan los beneficios de sus ventas. Sólo la atención sanitaria a cuatro patologías relacionadas con el consumo de tabaco (cánceres, enfermedades respiratorias, cardiovasculares y cerebrales) suponen un coste de 22.500 millones de pts. anuales. El coste estimado por las enfermedades sanitarias llega a los 60.000 millones de pts.; 33.156 millones corresponden a la atención sanitaria y asistencia hospitalaria (22.352 de gastos hospitalarios más 10.784 en prescripción farmacéutica, pruebas diagnósticas y atención primaria). Ésta es la cuantía que demanda el Gobierno autonómico (los andaluces aportan más de 120.000 millones de pts. en impuestos, que van a parar a las arcas de Hacienda). Actualmente se están realizando informes jurídicos, se cuenta con más de trescientos enfermos que colaborarán como testigos, se analizan 32 marcas de cigarrillos...

Hay quien dice que por qué las tabaqueras deben pagar el gasto que provocan si, por ejemplo, el culpable de un accidente de circulación no siempre es la empresa automovilística que fabricó el coche (y, en este sentido, por qué no se demanda a los fabricantes de pistolas o a los que elaboran productos que contienen colesterol). Muchos consideran que no hay que eximir a los ciudadanos de las responsabilidades que contraen con el ejercicio de su libertad.

\section{CAMBIOS LEGISLATIVOS}

El 15 de junio de 2001 el Parlamento Europeo aprobó la directiva sobre fabricación, presentación y venta de los productos del tabaco, norma que reduce los límites máximos de nicotina ( $1 \mathrm{mg}$. por cigarrillo), de alquitrán (de 12 a $10 \mathrm{mg}$.) y de monóxido de carbono (10 mg.) y obliga a las tabacaleras a especificar -a partir del 2004- los aditivos que utilizan en su elaboración y remitir anualmente a los Estados los detalles de los productos empleados, a los cuales corresponderá hacer pública esta información. Para entonces, la Comisión Europea deberá haber elaborado una lista de productos autoriza- 
dos. Los términos 'suave', 'light', 'ultralight', 'mild' (suave), 'bajo en nicotina', 'bajo en alquitrán'... y otros semejantes no podrán utilizarse, ni tampoco en la denominación de la marca que dé a entender a los usuarios que su producto es menos dañino (v.gr., las actuales Marlboro Lights o Camel Lights).

El cigarrillo 'light' (el 17\% de las ventas de cigarrillos rubios en España son "suaves", porcentaje inferior al del resto de Europa) nació a raíz de los informes sanitarios que en los sesenta y principios de los setenta señalaban la peligrosidad del tabaco. Las tabaqueras pusieron en marcha alternativas para hacerlo más saludable: cigarros con menos alquitrán y nicotina, es decir, con "más salud». Expertos rechazan que esta forma de fumar sea menos peligrosa: en muchos casos, la persona que se pasa al tabaco suave fuma de manera distinta, con caladas más profundas para llegar a la cantidad habitual de nicotina, apareciendo nuevos riesgos. Además, hay cánceres (de vejiga, laringe, páncreas...) que dependen también de otras sustancias, no sólo de la nicotina y el alquitrán.

La directiva fija, en cuanto a su presentación, que las cajetillas llevarán alternativamente las leyendas: 'Fumar mata', 'Fumar puede matar' o 'Fumar daña gravemente su salud y las de las personas que están a su alrededor' (otros como 'Fumar crea adicción' se rechazaron por ser menos contundentes), ocupando un tamaño que se eleva del $4 \%$ actual al 30\% en la cara anterior (32\% o $35 \%$ si se hace en dos o tres idiomas). En la parte posterior, habrá otras advertencias más concretas (riesgo de contraer algún cáncer, etc.), sin que se aceptaran otras propuestas más directas del tipo: 'Fumar provoca impotencia', 'Fumar reduce la fertilidad' o 'Fumar es un suicidio'. Sobre la posibilidad de emplear fotografías, la directiva deja libertad a los Estados para su uso, dado que la Comisión Europea estima que obligar la impresión de fotografías choca con las normas de mercado interior. La ministra de Sanidad española señaló recientemente que no se apuraría el plazo de septiembre de 2003 para la inclusión de las nuevas leyendas, sino que se impondrían de manera inmediata, y se decantó por la de 'Fumar mata'. Según el comunicado oficial de la Comisaría de Consumidores, esperan que con la nueva ley "el número de fumadores en la Unión Europea pase de un tercio a un quinto de la población comunitaria».

En cuanto a la publicidad del tabaco, la directiva que presentó la Comisión Europea para sustituir a la de 1998 -anulada en octubre de 2000 por el Tribunal de Luxemburgo- y que debe examinar el Parlamento Europeo y el Consejo de Ministros (puede entrar en vigor en 2004) propone prohibirla en prensa (excepto en revistas especializadas dirigidas a los profesionales), emisoras de radio e Internet -se perseguirían los banners de publicidad de tabaco- añadiéndose a la prohibición en televisión, que existe desde 1989. Como decíamos anteriormente, se renuncia a prohibirla en artículos relacionados con el tabaco, como los ceniceros (el Tribunal consideró que obstaculizaba el mercado interior), ni en cines (al no ser un producto transfronterizo), ni carteles o vallas callejeras. 
Tampoco patrocinará acontecimientos deportivos en los que participe o esté involucrado más de un país de la Unión (sí en los locales o nacionales), ni patrocinios de otra índole, como la distribución gratuita de tabaco. Ahora mismo, España tiene una de las normativas más permisivas en el ámbito de la publicidad del tabaco. Aunque está prohibida en televisión, han sido numerosos los anuncios de productos relacionados con marcas de tabaco y sólo se prohibe donde lo está la venta y el consumo de tabaco (hospitales, centros educativos y locales comerciales).

Otra línea de trabajo se centra en el aumento de espacios libres de tabaco. En España, un Real Decreto de 1998 prohibió fumar en los espacios públicos y se amplió en 1999 a los medios de transporte. Esta norma regula los lugares donde no se puede fumar: ascensores, colegios, hospitales, locales comerciales cerrados, salas públicas, transportes públicos y salas con ventanillas de atención al público, además de los lugares de trabajo cuando hay embarazadas. El Gobierno trabaja en una nueva normativa que lo prohibiría en los centros de trabajo, para lo que las empresas reservarían una sala para los fumadores. Como con otras medidas, unos se muestran a favor y otros en contra (para éstos, sería una discriminación), de difícil aplicación en las empresas pequeñas. En otros países de la Unión Europea ya lo está, por lo que deben ir a una sala especial o salir a la calle para fumar. Por cierto, diversas experiencias realizadas en nuestro país prohibiéndolo en los lugares habituales de trabajo han conseguido que muchos trabajadores hayan dejado el hábito. Según una encuesta de la Asociación Española Contra el Cáncer realizada a los responsables de casi un millar de compañías, sólo el $14 \%$ de las empresas siguen alguna política antitabáquica, pero el $90 \%$ considera adecuado regular su consumo en el trabajo. Mientras que las multinacionales y grandes empresas se muestran más receptivas, un $26 \%$ de los empleados eran reticentes y contrarios.

\section{LA REALIDAD DEL TABACO}

Recapitulando lo expuesto hasta ahora, podemos afirmar que el consumo de tabaco es la primera causa de muerte prematura, y evitable en las sociedades industrializadas, el principal problema de salud pública susceptible de prevención. Según algunas estadísticas, provoca el $20 \%$ de la mortalidad total. En nuestro país, un 13\% de las muertes que se producen están relacionadas con el tabaco (una de cada cincuenta directamente por el tabaquismo), aproximadamente 48.000 fallecimientos al año. Datos correspondientes a 1996 señalan que se registraron 600 millones de casos en todo el mundo con patologías de este tipo que dieron lugar a 2.800.000 muertes.

La OMS cifra unos 1.100 millones de personas fumadoras en el mundo y que tres millones y medio mueren anualmente por causas relacionadas con el tabaco (aproximadamente 10.000 al día); más de la mitad tienen lugar en 
los países industrializados (más de medio millón se producen en Europa). Las dolencias relacionadas con el tabaco serán la primera causa de muerte en los países en desarrollo en los próximos veinte años. Hacia el 2.020 el número de fallecimientos llegará a los 10 millones por año, de los que el $70 \%$ se producirán en los países en desarrollo (la fuerte penetración de la publicidad de las tabacaleras y los grandes ingresos que supone para las economías pobres -a través de los impuestos- ayudarán a ampliar estos efectos). En ese momento, producirá más muertes que la suma de las producidas por SIDA, tuberculosis, mortalidad prenatal, suicidios, homicidios, incendios, drogas ilegales y accidentes de circulación (Peto et al., 1994). Concretando más, el Informe Sobre la Salud en el Mundo 1999, realizado por la OMS, dice que de los 6.000 millones de habitantes en el mundo, casi 500 millones morirían a causa del tabaco si se mantienen las actuales pautas de consumo. Hasta el 2.024, morirán prematuramente a causa del tabaco unos 146 millones de personas, y desde el 2.025 al 2.050 , otros 300 millones.

Según el Comité de Oncólogos de la Comunidad Europea, una de cada tres muertes prematuras entre los 35 y 65 años tienen su causa en el consumo de tabaco. Otro dato significativo podemos encontrarlo en el Tobacco Alert, uno de los Boletines de la OMS: cada tres segundos muere una persona por causa del tabaco. El $90 \%$ de las muertes por cáncer de pulmón, el $75 \%$ por bronquitis crónica, el $25 \%$ de las causadas por problemas y enfermedades del corazón. No obstante, hoy en día se producen más muertes por enfermedades vasculares y coronarias que por cáncer de pulmón. El tumor pulmonar es la enfermedad oncológica más usual en los hombres, con un porcentaje aproximado del $20 \%$, y el segundo en el caso de las mujeres (con un 18\%), sólo superado por el cáncer de mama.

La mortalidad coronaria entre los fumadores es un 70\% superior a la de los no fumadores, y un año después de dejar de fumar, el riesgo de mortalidad coronaria disminuye un $\mathbf{5 0} \%$. Puede afirmarse que los fumadores tienen una tasa de mortalidad global (y coronaria) de casi el doble que los no fumadores. El riesgo de padecer ciertas enfermedades, la disminución de la esperanza de vida, etc. son otros efectos en los fumadores. También se estudia su impacto biológico en la fertilidad y, según recientes estudios, fumar -sobre todo si son más de diez cigarrillos al día- provoca elevados riesgos para el embarazo o para el niño de corta edad, entre ellos, la mayor probabilidad de que:

- el embrión se implante en un lugar distinto de la cavidad uterina;

- aborto espontáneo durante los primeros meses;

- la placenta resulte previa (colocada más abajo);

- gestosis;

- parto antes de tiempo; 
- el recién nacido pese menos de lo normal (200-300 grs.), menor longitud $(2-3 \mathrm{cms}$.) y circunferencia craneal inferior a la media $(1 \mathrm{~cm}$. aproximadamente).

En este sentido, Ponce, Muriel y Gómez de Terreros (1997:25) dicen que «el consumo de tabaco es probablemente el fenómeno más preocupante con relación a los hábitos nocivos para la madre y el niño». Un informe publicado recientemente en la Journal of the National Cancer Institute, por el equipo dirigido por el profesor Stephen Hecht (con investigadores de las Universidades Heinrich Heine de Dusseldorf y de Minnesota), señala que se ha encontrado un agente cancerígeno derivado químico de la nicotina en la primera muestra de orina de 22 hijos de un total de 31 recién nacidos de madres fumadoras (transmitido por éstas a los menores a través de la placenta). Además, los hijos de fumadoras están más expuestos a las alergias y, una vez nacido, el pequeño expuesto al humo corre un riesgo cuatro veces superior de coger infecciones bacterianas o víricas debido a la mayor irritación de las vías respiratorias altas y menor actividad de su sistema inmunitario.

En los últimos años, ha descendido su consumo entre los hombres y aumentado en las mujeres. Pronto serán visibles sus consecuencias (según diversos estudios, los pulmones de las mujeres son más sensibles a los efectos del tabaco debido a que los bronquios son más pequeños, tienen menor capacidad y mayor facilidad para obstruirse). Se han incrementado los ex-fumadores y son menos los grandes fumadores. El tabaco afecta a todos, al fumador activo y al fumador pasivo. Los males de los fumadores son competencia de todos, y no sólo de aquéllos. Costear los gastos sanitarios, las bajas laborales (los fumadores presentan mayores índices de absentismo en el trabajo y se registran más bajas laborales definitivas a edades más tempranas) corresponde al conjunto de la sociedad. Y se empieza a fumar muy pronto. El primero de los diez puntos para un programa de control del tabaquismo de la OMS con motivo del Día Mundial Sin Tabaco dice: Protección para que los niños no se conviertan en adictos al tabaco.

Queda claro que, por lo tanto, el tabaco es un producto nocivo y activo, que crea una adicción física y psíquica. Si bien la mitad de los fumadores manifiestan que quieren dejar este hábito, sólo el $5 \%$ lo hace espontáneamente y otro $10 \%$ lo consigue después de recibir consejo médico o por otro mecanismo similar. «El tabaquismo es la principal causa prevenible de enfermedad y muerte en las sociedades desarrolladas. El tabaco es el agente medioambiental más importante causante de patología humana, no sólo a los fumadores, sino también a quienes involuntariamente respiran el humo en ambientes cerrados. Nos encontramos ante una auténtica pandemia mundial de enorme magnitud y ante uno de los problemas más graves en Salud Pública de nuestro entorno; estamos ante un fenómeno evidente de drogadicción, tal como lo definió la OMS en 1979" (Galiano, Solbes y Gozalo, 1996). 
El abordaje preventivo, a nivel general o poblacional, se basa en ofrecer una correcta información sobre el tabaco y sus consecuencias, programas de educación para la salud, medidas y disposiciones legislativas al efecto, potenciar el papel modélico de los profesionales, educadores, políticos... Su consumo está determinado por la disponibilidad, la legalidad y el precio. Otras medidas pasarían por aumentar los precios, restringir su uso y venta, suprimir la publicidad, etc. Los programas de terapia individual para eliminar el hábito deberían encuadrarse en programas antitabaco destinados a la población en general para una mayor eficiencia y eficacia. Un estudio editado por la OMS, que evalúa distintas experiencias y actividades de lucha contra el tabaco (Chollat-Traquet, 1998), destaca el recurso de los medios de comunicación, la educación en las escuelas, la intervención comunitaria, la participación de los agentes implicados como educadores... La OMS (1979) señaló las metas que debían marcarse desde una acción comunitaria:

- Reducir al máximo el número de jóvenes que empiezan a fumar, así como retrasar todo lo posible su iniciación.

- Reducir el número de fumadores, con la orientación y apoyo necesario para que lo dejen.

- Procurar que los que no puedan dejar este hábito disminuyan la inhalación de sustancias nocivas del tabaco.

- Proteger los derechos de los no fumadores.

- Crear un ambiente social negativo ante su consumo.

Hoy en día, estos objetivos se mantienen. Entre los aspectos prioritarios para las políticas de prevención en España, según el Comité Nacional de Prevención del Tabaquismo, destacan:

- Dispositivos de ayuda para los que deseen dejarlo.

- Revisión de la política fiscal para reducir el acceso.

- Regulación de la publicidad y otras formas de promoción.

- Regulación de la venta, especialmente a menores de 18 años.

- Regulación del contenido de los cigarrillos.

- Más información al consumidor de los productos.

- Ampliación real de los espacios públicos cerrados sin humo.

Como complemento de lo anterior, podemos citar algunas «ideas de parti- 
da» para un Programa de Prevención de Tabaco (Ortega y Camacho, 1993:15):

- Está demostrado científicamente que es nocivo para la salud.

- El tabaquismo es un grave problema social, tanto por su extensión entre la población como por el deterioro de salud que supone.

- Al ser un gran problema de salud, debe contemplarse como tema de atención prioritario desde los estamentos sociales: sanitarios, educativos, políticos...

- Existe un factor de riesgo muy importante de cara a la introducción del individuo al consumo de esta droga, al estar incorporada a la vida cotidiana y a las costumbres sociales.

- Un gran porcentaje de fumadores inicia el hábito en edad escolar o en la adolescencia.

- Por ello, y desde una actitud de prevención, la escuela no debe permanecer al margen, sino que debe desarrollar programas de Educación para la Salud que capaciten al alumnado para la toma de decisiones conscientes y críticas y "aprenda a saber decir No".

Si numerosos estudios señalan que una gran mayoría de los fumadores declara que les gustaría dejarlo, pero no lo pueden hacer, la solución estaría en no probarlo (valga como ejemplo que en España más de un $60 \%$ de los fumadores han intentado dejarlo al menos una vez y distintos estudios señalan que una vez iniciada la conducta de fumar, resulta adictiva para el 95\%). Además, cada vez se entra en contacto más temprano con el tabaco, principalmente en la adolescencia (en España, cerca de la mitad de los jóvenes lo han probado antes de los 16 años y el $26,4 \%$ de los que tienen entre 15 y 18 años fuman regularmente).

La última Encuesta sobre Drogas a la Población Escolar 2000 del Plan Nacional sobre Drogas, presentada en julio de 2001 y con datos de 20.450 encuestas pasadas en noviembre de 2000 , recoge que el $34,4 \%$ de los escolares españoles de entre 14 y 18 años lo han probado, siendo la droga que se empieza a consumir a una edad más temprana, a los 13,2 años, seguida por el alcohol a los 13,6 y el cannabis a los 14,8. Después de tres años de descender la edad de inicio, en el 2000 se estabilizó para esas tres sustancias (sí disminuye en las demás, como alucinógenos, anfetaminas, éxtasis o cocaína, aunque el primer contacto es todavía más tardío). Según la Encuesta, el $30,5 \%$ había fumado en el último mes y un $5 \%$ era ya ex-fumador. Las chicas consumen con mayor frecuencia tabaco $(34 \%-24 \%)$, tranquilizantes y alcohol que los varones, aunque en menores cantidades. Casi la mitad consume entre uno y cinco cigarrillos al día. Considerando que casi la totalidad de 
niños y jóvenes están escolarizados obligatoriamente hasta los 16 años, no puede ponerse en duda que los centros educativos son el lugar idóneo para plantear temas de educación para la salud en relación con el tabaco.

\section{EDUCACIÓN PARA LA SALUD Y SU TRATAMIENTO "TRANSVERSAL"}

Las líneas siguientes, como podrá comprobarse, no sólo tienen validez en el campo de la educación formal, sino que pueden aplicarse en otras experiencias educativas de distinto carácter y naturaleza, si bien queda claro que el tabaco es uno de los contenidos que aborda la Educación para la Salud (EpS), una de las Materias Transversales. Sin profundizar en este aspecto, que no es nuestro objetivo, sí quisiera reafirmar la importancia de su inclusión como tal. En otros momentos ya he expresado el impulso que ha supuesto su consideración "curricular» (Morón Marchena, 1995a; 1995b; 1998b). Según la LOGSE, la EpS se define como una enseñanza que debe estar presente en las diversas áreas educativas, en el conjunto del currículo y en todo el proceso educativo, en el propio proyecto educativo de la escuela. Debe ser un instrumento de concienciación acerca del propio valor de la salud para la vida. Se extiende más allá de las áreas de conocimiento, al campo de los valores y actitudes. Los Temas Transversales estimados por cada Comunidad Autónoma con transferencias en el ámbito educativo varían, aunque se basan fundamentalmente en el listado que consideró el M.E.C. En todos los casos se incluye la EpS como transversal, algunas veces junto a la Educación Sexual o Educación para el Consumo. Los contenidos relacionados con las Drogas se recogen dentro de la EpS, aunque a veces se explicitan como una transversal con suficiente peso como para ser tratada más específicamente. Tal como recoge los DCB, la EpS cumple las características para ser considerada materia transversal, ya que:

- responde a problemas que actualmente tiene planteada la sociedad;

- abarca contenidos de varias disciplinas y sólo pueden abordarse desde su complementariedad;

- no puede plantearse como programas paralelos al resto del currículum, ya que deben ser asumidos por el conjunto de los enseñantes y estar presentes en gran parte de los contenidos escolares;

- transciende del estricto marco curricular e impregna la totalidad de las actividades del centro; y

- está estrechamente relacionada con la transmisión de valores a través del currículum oculto.

Por lo tanto, abordar el tabaco en el aula es uno de los contenidos de la EpS, inmerso en un contexto más amplio de Promoción de la Salud. Existe 
un acuerdo general entre todos los países europeos sobre los contenidos que deben estar presentes en un programa escolar de EpS. Entre ellos se incluye el «Uso y abuso de medicamentos y drogas (legales e ilegales)», con el tabaco, el alcohol y las drogas ilegales (Young y Williams, 1989:15). La prevención del tabaquismo desde la escuela puede plantearse desde una filosofía general de la actuación educativa o desde el marco concreto de la EpS, pero una adecuada prevención exige la interacción en el Proyecto Educativo de Centro a través de sus diversos elementos, tales como el Plan de Acción Tutorial, Plan de Formación del Centro, Reglamento de Organización y Funcionamiento, objetivos educativos, relación ente todos los agentes implicados, etc. así como en el Proyecto Curricular de Etapa (González García, 1998). La Educación sobre Drogas suele plantear objetivos en tres líneas (Comisionado para la Droga, 1989:12-13):

- Objetivos de conducta: la total abstinencia, reducir los niveles de consumo, mantener ciertos niveles de consumo, modificar las situaciones en las que se fuma, etc.

- Objetivos afectivos: incrementar los sentimientos o actitudes negativas, disminuir las positivas, promover una actitud de aceptación hacia medidas sociales y políticas contra las drogas, modificar valores...

- Objetivos informativos: elevar el nivel de conocimiento relacionado con la naturaleza de las drogas, su química y propiedades farmacológicas y conductuales.

Estos objetivos se resumirían en: a) distribuir una información de base sobre el tema a niños, padres, educadores y comunidad en general; b) promover una mejor comprensión de los problemas causados por las drogas; c) $\mathrm{y}$ proponer a los jóvenes soluciones y alternativas para responder a sus necesidades y aspiraciones.

En general, las hipótesis en las que se basan las actuaciones educativas son la posibilidad de modificar el comportamiento de los sujetos, si se modifica previamente su comportamiento, las actitudes y los sistemas de valores. En el Manual de Formación para el Profesorado y otros agentes educativos "La Educación para la Salud en el ámbito educativo" (OMS y Comisión de la Unión Europea), se incluye el tabaco dentro del tema referido a la «Educación sobre las Drogas". Se le presta una atención especial, por su carácter "legal", por su uso tan extendido, que además sirve de puente hacia otras drogas «ilegales». Resumamos seguidamente los principales aspectos que se recogen en el mismo (Young, 1995:166-172).

Tratar el tabaco como uno de los contenidos a abordar en EpS requiere considerar el porqué, cómo y cuándo se consume. El profesorado debe ser consciente del problema, entender sobre el uso del mismo y estar familiarizado con técnicas de enseñanza que ayuden a los jóvenes a adoptar las deci- 
siones oportunas. Es una parte más de la EpS escolar, siendo éste un programa que trata de todos los aspectos de salud personal, familiar y comunitaria, integrada en todo el sistema de valores de la escuela y el entorno familiar y social, fomentando la construcción de la autoestima, abordando las relaciones personales y facilitando la adopción de decisiones informadas como habilidades para los jóvenes. Para ello se requiere una adecuada planificación, incluyendo materiales y técnicas, con la implicación de todo el profesorado y olvidando los enfoques alarmistas y sensacionalistas. Los contenidos a tratar deben incluir información sobre hechos y conceptos relacionados con el tabaco, sus efectos, aspectos legales de su uso, proceso de adicción, alternativas a su consumo... Más que un conjunto de conocimientos académicos, se trata de información para poder tomar decisiones, poner en práctica habilidades sociales y ofrecer modelos de comportamiento. El método debe ser eminentemente participativo, con un aprendizaje experiencial y activo, con dinámicas de grupo para desarrollar relaciones personales (aumentando la autoestima y confianza).

Coincidiendo con lo apuntado anteriormente, la educación sobre el tabaco es un proceso que implica: informar al alumnado sobre la composición y sus efectos; tener en consideración los efectos sociales, mentales y físicos de su consumo; y el desarrollo de habilidades para adoptar decisiones adecuadas a las situaciones en las que se fuma. El Manual señala que la educación debe comenzar lo más pronto posible, pues en las primeras fases del uso de las drogas se aprenden los modelos de consumo, que pueden llegar a ser significativos para determinar las posteriores formas de uso. Y más teniendo en cuenta que es muy asequible a los jóvenes: lo pueden comprar fácilmente sin tener la edad legal para ello (v.gr., a través de las máquinas expendedoras automáticas). Son varios los aspectos que influyen en el desarrollo del hábito de fumar:

- los factores personales (los jóvenes lo asocian con una parte importante de su propia imagen);

- las influencias de iguales y amistades (un elemento socializador, de identidad al grupo, para introducirse en algún círculo);

- los modelos paternos y maternos: los padres fumadores son un aspecto facilitador (ampliable a otros posibles modelos a imitar, como son los docentes);

- la influencia de los medios de comunicación social y el entorno (que estimulan una actitud favorable para consumir tabaco, vendiendo valores e imágenes de deporte y triunfo social vinculado a las marcas);

- también influyen los conocimientos, valores, actitudes y objetivos relacionados con el fumar que se enseñan para prevenir su posterior consumo. 
Las actitudes y los valores relacionados con el uso del tabaco se forman en una edad temprana, siendo a través de la observación y/o la experimentación como mejor se aprende. Abordarlo desde un modelo únicamente informativo-preventivo, basado en informar sobre los efectos y características de aquél, no basta para tener el éxito asegurado. Se puede tener bastante información, pero adquirir o mantener hábitos no saludables. De ahí el interés por modelos combinados que incluyan programas informativo-preventivos con modelos de actitudes (Delgado, Pablos y Sánchez, 1996:61-65). "La influencia de los factores ambientales es tan obvia que a veces no se les da importancia, y se piensa que con una mera información sobre el tema en cuestión es suficiente. En muchas ocasiones se supone que el solo conocimiento de los daños que produce el humo del tabaco es suficiente para que el adolescente deje de fumar. Ésta es una idea totalmente errónea, ya que está presionado por una multiplicidad de actores sobre los que a duras penas puede mantenerse firme, motivo por el que se necesita apoyo desde los más diversos ángulos" (Llorent, 1990:65-66). De hecho, no se da siempre una relación estadística positiva entre conocimientos sobre drogas y su no consumo (valga el ejemplo de los docentes, médicos y enfermeros que fuman). Tampoco se da una relación casual entre cambios de conocimiento y variaciones en los hábitos de consumo. Muchos autores señalan que a los jóvenes les son más efectivos mensajes del tipo 'el tabaco ensucia los dientes', 'amarillentan las manos'... que los típicos 'perjudica la salud'.

\section{A MODO DE COROLARIO. EFECTIVAMENTE, UN RETO SOCIOPEDAGÓGICO}

Con todo lo expuesto, se responde de modo afirmativo a la pregunta que nos hacíamos al principio. El tabaco, esta droga «especial», es una cuestión importante a la que debe responder la EpS, superando el ámbito estrictamente sanitario y/o escolar. Es un reto comunitario, social. García Mínguez (1997) dice que el tabaquismo, como la adicción a otras drogas, se reconduce por canales sociales... Sin duda un problema de envergadura en el que todos y todas estamos implicados. Concluimos sirviéndonos de las palabras de Víctor López y Rafael Manzanera en el Prólogo de una reciente obra sobre el tabaquismo (Villalbí y Ariza, 2000) que enfocan la compleja realidad a la que nos enfrentamos. "Es en España la primera causa aislada de enfermedad evitable, invalidez y muerte prematura, y necesita un abordaje integrado, en el marco de una política integral de salud: reducir la cuestión al libre albedrío personal sería una dejación política y un cinismo inaceptable. En efecto, si el tabaquismo es un problema mayor de salud (a diferencia de hace un siglo) es por el formidable negocio que lo propicia y por la naturaleza adictiva de la nicotina".

En este contexto, es una situación singular: se trata del único producto de consumo capaz de producir lesiones y hasta la muerte a muchos de sus usuarios al ser utilizado estrictamente conforme a las indicaciones del fabricante. 
Esta realidad lleva a la industria tabaquera a la posición extrema de negar lo evidente, pues cualquier otra postura la confrontaría a la asunción de sus responsabilidades y cuestionaría la esencia de su negocio. En un contexto democrático, y ante un producto utilizado por una porción no mayoritaria pero relevante de la población, no se plantea su prohibición. Pero sí creemos necesaria una regulación rigurosa de los productos de tabaco, de su comercio, promoción y consumo, en defensa de los bienes públicos, como la salud de la población y la protección de los menores. Por ello, la prevención del tabaquismo no puede basarse sólo en estrategias centradas en los servicios sanitarios. El rol de los profesionales asistenciales de ayudar a los fumadores a romper su adición es crucial para muchas personas. Pero la prevención supera los límites de los servicios clínicos. Una política preventiva frente al tabaco implica el desarrollo de estrategias inspiradas en las ciencias políticas y sociales, capaces de comprender y usar los medios de comunicación. En realidad, perfila una nueva agenda de salud pública y de promoción de salud, en la que debe destacarse el papel fundamental del ámbito educativo formal, no formal e informal.

\section{BIBLIOGRAFÍA}

BARRUECO FERRERO, M. (1998): "Prevención del Tabaquismo en niños, adolescentes y jóvenes", en Romero, P.J., León, M.J. y Gallardo, M.A.: Educar para la Salud: Mejor sin humos. Granada: Grupo Editorial Universitario, pp. 63-68.

BECOÑA IGLESIAS, E.; GALEGO, P. y LORENZO, M.C. (1988): El tabaco y su abandono. Santiago de Compostela: Plan Autonómico sobre Drogodependencias-Conselleria de Sanidade.

COMISIONADO PARA LA DROGA (1989): La intervención educativa ante las drogas. Cuaderno de orientación para educadores. Sevilla: Consejería de Asuntos Sociales.

CHOLLAT-TRAQUET, C. (1998): Evaluación de las actividades de lucha contra el tabaco: Experiencias y principios orientadores: Organización Mundial de la Salud.

DE LA ROSA ACOSTA, B. (1995): «La Educación para la Salud, un nuevo reto educativo", en Morón Marchena, J.A. (Dir.): Educación para la Salud en el ámbito comunitario. Sevilla: Diputación de Sevilla, pp. 27-44.

DE VRIES, H. et al. (1999): European smoking prevention framework approach. Las Palmas: European Conference on Tobacco or Health (CD-ROM).

DELGADO, E.; PABLOS, M.L. y SÁNCHEZ, D. (1996): Programa de Prevención de Drogodependencias (Tabaco y Alcohol) al finalizar la Educación Primaria. Sevilla: Comisionado para la Droga (Junta de Andalucía).

DIRECCIÓN GENERAL DE PREVENCIÓN Y PROMOCIÓN DE LA SALUD (1994): Actualizaciones sobre el Tabaco. Documento Técnico de Salud Pública $n .^{\circ}$ 6. Madrid: Consejería de Salud de la Comunidad de Madrid. EDIS (1997): Los andaluces ante las Drogas $V$. Sevilla: Comisionado para la Droga (Junta de Andalucía). 
GALIANO, J.J.; SOLBES, A. y GOZALO, J.L. (1996): "Tabaquismo-Prevención», en Macías, B.E. y Arocha, J.L. (Dirs.): Salud Pública y Educación para la Salud. Las Palmas: ICEPSS, pp. 455-468.

GONZÁLEZ ENRÍQUEZ, J. et alt. (1989): «Muertes atribuibles al consumo de tabaco en España». Medicina Clínica (Barcelona), núm. 92, pp. 15-18.

GONZÁLEZ GARCÍA, F. (1998): «Salud y Tabaco: Contenidos Curriculares Transversales», en Romero, P.J.; León, M.J. y Gallardo, M.A.: Educar para la Salud: Mejor sin humos. Granada: Grupo Editorial Universitario, pp.29-34.

GONZÁLEZ HERNÁNDEZ, A. y GARCÍA MARTÍNEZ, A. (1998): “ $¿$ Es socialmente útil la Educación para la Salud?», en Morón Marchena, J.A. (Dir.): Educación para la Salud. Fundamentos y Metodologia. Sevilla: Ayuntamiento de Dos Hermanas, pp. 27-44.

IRANDA, A.; SILERO, M.I. y ANDRADAS, E. (2000): «Estudio descriptivo Tabaquismo en el profesorado de Primaria y Secundaria del Área IX de la Comunidad de Madrid", en V Conferencia Europea de Promoción de la Salud y de Educación para la Salud "Hacia una Europa Saludable en el año 2.010\%. Madrid: Asociación de Educación para la Salud, pág. 69.

JESSER, R. y JESSER, S. (1977): Problem behavior and psychosocial development. New York: Academic Press.

LLORENT BEDMAR, V. (1990): Factores sociales que inciden en el consumo de tabaco: Estudio comparado y Bases para una Educación Preventiva. Sevilla: Consejería de Salud.

MENDOZA, R.; VILLARRASA, A. y FERRER, X. (1986): La educación sobre las drogas en el Ciclo Superior de $E G B$. Madrid: MEC

MORÓN MARCHENA, J.A. (1995a): "Reflexiones en torno a la Educación para la Salud. Un reto actual", en Educación para la Salud: un reto para todos. Sevilla: Ayuntamiento de Dos Hermanas, pp. 124-130.

MORÓN MARCHENA, J.A. (1995b): «La salud en la trilogía comunidad, educación y sanidad", en Educación para la Salud en el ámbito comunitario. Sevilla: Diputación de Sevilla, pp. 45-56.

MORÓN MARCHENA, J.A. (1998a): "Tabaco y Educación para la Salud", en Romero, P. J., León, M. J. y Gallardo, M.A.: Educar para la Salud: Mejor sin humos. Granada: Grupo Editorial Universitario, pp. 47-62.

MORÓN MARCHENA, J.A. (1998b): Nuevas demandas de la sociedad actual: Educación para la Salud y Educación para el Consumo. Sevilla: GIPDA-Edit. Kronos.

MORÓN MARCHENA, J.A. (1999): «Educación para la Salud y Tabaco: Un reto sociopedagógicon, en: Educación para la Salud y Municipio. Sevilla: Ayto. de Dos Hermanas y Consejería de Salud de la Junta de Andalucía, 145-175.

ORGANIZACIÓN MUNDIAL DE LA SALUD (1979): Europa sin Tabaco. Plan de Acción a cinco años. Madrid: Ministerio de Sanidad-Oficina Regional Europea.

ORTEGA MURCIA, J. et al. (1993): Tabaquismo y alcoholismo: un problema social (Educación para la Salud). Madrid: Ministerio de Sanidad y Consumo-M.E.C.

PETO, R. et al. (1994): Mortality from smoking in developed countries. 19502000. Oxford: University Press.

PNUD (1998): Informe sobre el desarrollo humano 1998. Madrid: ONU-Ediciones Mundi-Prensa.

PONCE, J.A.; MURIEL, R. y GÓMEZ DE TERREROS, I. (Eds.) (1997): Salud, Infancia, Adolescencia, Sociedad. Sevilla: Asociación Española de Pediatría.

ROONEY, J.F. y VILLAHOZ, J. (1995): $E l$ consumo de drogas entre los estudiantes de 10 a 18 años. Sevilla: Comisionado para la Droga. 
SAINZ MARTÍN, M. (1997): Qué hacer con la salud. Madrid: I.S.E.-Cuadernos de Acción Cultural.

SOLANO REINA, S. (1997): "Consumo mundial de Tabaco. Datos relacionados con España». Prevención del tabaquismo. SEPAR, 8, 12-17.

SOLANO REINA, S. et al. (2000): "Papel de los profesionales de la salud ante el hábito tabáquico», en Actualizaciones sobre el tratamiento del tabaquismo. Madrid: Dirección General de Salud Pública de la Comunidad de Madrid, 7-18.

TORRES, M.A. (1990): «El consumo de las drogas en la población escolar", en Escámez, J.: Drogas y escuela. Madrid: Dykinson, pp. 35-48.
VEGA FUENTE, A. (1993): La acción social ante las drogas: Propuestas de intervención socioeducativa. Madrid: Narcea.

VILLALBI, J. R. y ARIZA, C. (2000): $E l$ tabaquismo en España: situación actual y perspectivas para el movimiento de prevención. Barcelona: SESPAS.

YOUNG, I. (Coord.) (1995): Promoción de la Salud en la juventud europea. La Educación para la Salud en el ámbito educativo. OMS-MEC.

YOUNG, I. y WILLIAMS, T. (Eds.) (1989): La escuela saludable. Edimburgo: OMS-Grupo Escocés de Educación para la Salud.

\section{RESUMEN}

El tabaco es la primera causa aislada de mortalidad prematura (de enfermedad evitable, invalidez y muerte prematura). En el marco de la Educación para la Salud, es una cuestión que debe tratarse desde un planteamiento de carácter sociopedagógico -como tantos otros ámbitos de la Promoción de la Salud-, por cuanto: a) su consumo tiene efectos muy periudiciales para la salud, pero debe abordarse desde una perspectiva social, superando la meramente médica. Conlleva aspectos económicos, fiscales, legislativos... b) Por otro lado, cada vez somos más conscientes de que la educación es el "arma" más importante para enfrentarse de manera preventiva al consumo del tabaco.

Desde esta visión, la prevención del tabaco es, pues, una tarea eminentemente social y comunitaria, y la educación es la herramienta primordial para afrontar este tema. Pero para conocer la realidad del tabaco, hay que conocer todos los aspectos que lo rodean: relevancia e incidencia social, efectos, consecuencias para la salud, datos estadísticos, legislación, fiscalidad, el marco escolar, etc. El artículo pretende, desde un ángulo informativo y divulgativo, acercarmos a estas cuestiones.

Palabras clave: Educación para la Salud, Promoción de la Salud, Prevención de Drogas, Tabaco, Consumo de Tabaco, Fumadores/as, Fumador Pasivo, Prevención del tabaquismo, Programas Educativos. 


\section{ABSTRACT}

Tobacco is the first isolated reason (on itself) of premature mortality (avoidable disease, invality and premature death). Within the frame health education there is a question to be dealt with from a sociopedagógical approach-as so many other areas in promotion of health-since: a) its consumption has very harmful effects on health, therefore it must be approached from a social perspective, overcoming the merely medical one. It implies economic, fiscal, legislative aspects... b) On the other hand, time after time we are more and more conscious that education is the most important "weapon» to intervene, in a preventive way, the consumption of tobacco

From this point of view, the prevention of tobacco consumption is, thus, mainly a social and community task, and education is the basic tool to confront this topic. But to know the reality of tobacco, it is necessary to know all aspects that gather around it: social relevancy and incidence, effects, consequences for health, statistical information, legislation, tax system, the school frame... The article tries, from an informative and divulgative point of view, to make us reflect about to these questions.

Key words: Health Promotion, Health Education, Drug Prevention, Tobacco, Tobacco Consumption, Smokers, Passive smoking, Smoking Prevention, Educational Programmes. 